\title{
An efficient method for optimal design of large-scale integrated chemical production sites with endogenous uncertainty
}

\author{
Sebastian Terrazas-Moreno, Ignacio E. Grossmann \\ Chemical Engineering Department \\ Carnegie Mellon University \\ Pittsburgh, PA 15213
}

John M. Wassick, Scott J. Bury, Naoko Akiya

The Dow Chemical Company

Midland, MI 48674

May 2011 


\begin{abstract}
Integrated sites are tightly interconnected networks of large-scale chemical processes. Given the large-scale network structure of these sites, disruptions in any of its nodes, or individual chemical processes, can propagate and disrupt the operation of the whole network. Random process failures that reduce or shut down production capacity are among the most common disruptions. The impact of such disruptive events can be mitigated by adding parallel units and/or intermediate storage. In this paper, we address the design of large-scale, integrated sites considering random process failures. In a previous work (Terrazas-Moreno et al., 2010), we proposed a novel mixed integer linear programming (MILP) model to maximize the average production capacity of an integrated site while minimizing the required capital investment. The present work deals with the solution of large-scale problem instances for which a strategy is proposed that consists of two elements. On one hand, we use Benders decomposition to overcome the combinatorial complexity of the MILP model. On the other hand, we exploit discrete-rate simulation tools to obtain a relevant reduced sample of failure scenarios or states. We first illustrate this strategy in a small example. Next, we address an industrial case study where we use a detailed simulation model to assess the quality of the design obtained from the MILP model.
\end{abstract}

Keywords: Integrated sites, process reliability, endogenous uncertainties, bi-criterion optimization, mixed-integer linear programming, Benders decomposition.

\title{
Introduction
}

The optimal design and operation of integrated production networks is a current and future opportunity in the chemical process industry. For instance, The Dow Chemical Company owns Texas Operations, an integrated site that manufactures $21 \%$ of the company's products sold globally (Wassick, 2009). BASF's site in Ludwigshafen is another example of a large integrated production system with over 200 production plants (BASF, 2010). Both of these sites began as smaller manufacturing facilities and grew in capacity and complexity over many decades. In contrast with the gradual integration of 
these heritage sites, recent strategic initiatives require the grassroots design of very large integrated process networks. The joint venture between Saudi Aramco and the Dow Chemical Company to construct and operate a world-scale chemical and plastic production complex in Saudi Arabia is an example of such an initiative (Dow, 2007).

These integrated sites feature different interconnected process networks. A failure event that reduces the production rate of any of the processes can propagate throughout the network. Some events are planned, for example, preventive maintenance of major plant components; the remaining failure events occur at random, requiring corrective maintenance. The focus of this work is on the second type of events, namely, failure modes that decrease production capacity and that occur at random times with random repair durations. The industrial significance of this problem is illustrated in a paper by Miller et al. (2006) from The Dow Chemical Company. These authors explain the benefit of designing reliability into manufacturing systems and illustrate the scope of the involvement of Reliability-Availability-Maintainability (RAM) teams during the design of large-scale manufacturing systems. A note on terminology; availability is the ratio of uptime to total time or the fraction of time the unit is producing product, reliability is the probability of a unit or piece of equipment being in an up state at a particular time.

To study these stochastic failures, computer simulations are commonly used to test the effect of design alternatives in the availability or effective capacity of integrated systems. This approach is applicable to an integrated chemical facility. As expected for an integrated system, the increasing number of design degrees of freedom and the increasing number of stochastic inputs quickly increases the difficulty and time to search the design space, requiring more computing and modeling resources. In addition, the successful ranking and selection of options is often challenging to do cleanly when the search space is large. We believe that mathematical programming techniques can be used in conjunction with process simulation tools to provide an efficient tool for improving the design of integrated sites by significantly reducing the design space. This new space can be then thoroughly explored and validated via simulation. Additional opportunities for applying optimization to the solution of the design, planning, and scheduling of integrated sites can be found in Wassick (2009). 
In our previous work (Terrazas-Moreno et al., 2010), we proposed a mixedinteger linear programming (MILP) model for the optimal design of integrated sites subject to random failures and random supply and demand. The optimization criteria were the maximization of the probability of meeting customer demands and the minimization of capital investment. To be an practical and useful tool for design teams, this challenging model must be solved efficiently. A literature review of optimization approaches for related problems, which includes the works by Davies and Swartz (2008), Pitikopoulos et al. (1996, 2001), and Straub and Grossmann (1993, 1990), can be found in that paper.

In the present paper, we propose a novel algorithm based on Benders decomposition (BD) (Benders, 1962) to solve large-scale instances of the MILP problem mentioned above. There is rich literature on the application of $\mathrm{BD}$ to the design of process systems under uncertainty. Most of these applications model uncertainty using a stochastic programming (SP) representation and apply variations of the BD algorithm. The standard decomposition technique is referred to as L-shaped decomposition (Van Slyke and Wets, 1969) in the stochastic programming literature. Straub and Grossmann (1993) proposed a nonlinear programming (NLP) model for maximizing the feasible operating region of a network with uncertain process parameters and used Generalized Benders Decomposition (GBD) (Geoffrion, 1972) to solve this problem. A similar approach was proposed by Pistikopoulos (1995) and applied by Ierapetritou et al. (1996) and Acevedo and Pistikopoulos (1998) as a general algorithmic technique for solving a class of problems defined as process design and operations under uncertainty. More recently, Liu et al. (2009) addressed the design of reliable transportation networks subject to unpredictable natural disasters using Generalized Benders Decomposition (GBD).

The work by Santoso et al. (2005) is related to the model and algorithm we present in this paper, although they deal with exogenous uncertainties as opposed to endogenous uncertainties in our case. The paper by Santoso et al. (2005) proposes a twostage stochastic programming (SP) model to optimize the design of a supply chain network under uncertainty. The authors consider uncertainty in parameters such as processing cost, raw material supply, finished product demand, and processing capacity of manufacturing facilities. These uncertain parameters are discretized in order to build 
scenarios with different combinations of parameter values. The resulting number of scenarios can be huge for realistic problem instances. The paper proposed an algorithm based on $\mathrm{BD}$ where the $1^{\text {st }}$ stage network design variables are considered complicating. An interesting feature of this paper is that Benders decomposition is enhanced with convergence accelerating techniques based on three ideas. The first is adding constraints besides the usual dual cuts to the master problem that can be derived as strengthened dual cuts or from constraints expressed in terms of variables in the master problem that were redundant and not included in the full space model. The second idea is a heuristic for finding good feasible solutions. The third is a trust region algorithm that prevents the master problem from oscillating wildly in the first iterations.

All of the implementations of BD and GBD mentioned above correspond to SP problems with exogenous uncertainties. That is, the stochastic process is independent of design decisions (Jonsbraten, 1998). If we exclude exogenous uncertainties like demand and raw material supply, then the source of remaining uncertainties are random process failures, which are dependent on design decisions. This implicitly assumes that when a unit is "up" that it operates at the design rates. The number and selection of parallel processing units in the network are not known a priori, i.e., they are decision variables. Since the only processes where random failures can occur are those that are selected from the superstructure, the realizations of uncertainties are also a function of the decision variables. This type of uncertainty, defined as endogenous, significantly increases the complexity of the problem as well as the computational resources required for solving it. This paper proposes a novel implementation of Benders decomposition for two-stage stochastic programming (SP) problems with endogenous uncertainties. The technique we propose partly overcomes the combinatorial explosion in problem size that occurs with non-anticipativity constraints required in this type of problems (Jonsbraten, 1998).

In the following sections, we present the problem of optimal design of integrated sites and its representation as a two-stage stochastic programming problem. Some important properties of the problem are given, and the decomposition approach that exploits these properties is presented. Finally, we test the methodology with two numerical examples, one of them being an industrial case study. As part of the solution of this case study, we analyze a scenario reduction technique, and we report the results of 
simulating the operation of the Pareto-optimal designs with a commercial simulation tool, ExtendSim ${ }^{\circledR}$ ((Imagine That, Inc., 2010).

\section{Problem statement}

In this section, we describe the problem of optimal design of highly available integrated sites.

The following list of given data, degrees of freedom, optimization criteria, and assumptions is reproduced with minor changes from Terrazas-Moreno et al. (2010).

\section{Given are:}

- The superstructure of an integrated site with allowable parallel production units in each plant and intermediate storage tanks.

- A set of materials that the plants consume and produce.

- Mass balance coefficients for all units in the superstructure.

- The maximum capacity that can be assigned to each unit in the integrated site.

- Maximum supply of raw materials and maximum demand of finished products.

- Number of failure modes, production rate loss as a result of each failure, and the time between failure (TBF) and time to repair (TTR) per failure mode, either as probability distributions or mean values (MTBF and MTTR, respectively).

- A cost function that relates design decisions with capital investment.

The problem is to determine:

- The number of production units for each plant.

- The capacity of each unit.

- Sizes of intermediate storage between plants.

- For each state, material flows between plants and rate of accumulation or depletion of material in storage.

The objective is to determine the set of Pareto-optimal solutions that:

- Maximize the average production rate at which the integrated site supplies chemicals to an external market.

- Minimize the capital investment. 
An interesting aspect of our approach is the development of a set of discrete states that correspond to all possible combinations of failure modes in the integrated site and a set of frequency and duration equations that allows us to calculate the mean residence time $m r t$ and frequency of encounters $f r$ of all possible states in the system, based only on the knowledge of the MTTR and MTTF of the units in the superstructure (Billinton and Allan, 1992).

The following assumptions and simplifications are made:

1. Random failures are independent events.

2. The cost function is represented by piece-wise linear approximations.

3. The production units in the plants are dedicated single product continuous processes.

Multiple failures in real integrated systems can be causally related. If this is the case, our first assumption will result in inaccurate statistical information of the discrete states described above. In practice, this is rare except at the equipment component level, which is at a level a detail finer than the failures considered in this work. and consequently most reliability simulation is carried out assuming independent failure modes. In this sense, the independence assumption made in this work will not add any more inaccuracy than what is regularly used in industrial simulations. Statistically uncorrelated events allow us to determine discrete state probabilities, residence times, frequency, etc. using standard frequency and duration calculations (Billinton and Allan, 1992). Using a piecewise linear approximation of the objective function preserves linearity in the problem. The number of piecewise functions can be increased or decreased depending on the level of precision required in cost calculations. Finally, the third assumption limits the applicability of the approach to systems that can be modeled as continuous dedicated plants. Multiproduct plants would require scheduling considerations and a system of multiple storage tanks. 


\section{Overview of solution strategy}

The solution approach we use in this paper integrates simulation and optimization tools. The simulation is built as a discrete rate model in ExtendSim software (Imagine That, Inc., 2010). In a discrete rate model, flow rates and tank levels are updated only when needed, by an internal Linear Programming (LP) solver at each discrete event. In our problem, events are failures and repairs that change the rate of flow of materials among plants and changes in tank level status (e.g. full, empty, high, low). The optimization step involves a Mixed-Integer Stochastic Programming representation of the integrated site and exploits state-of-the-art computational technology for solving Mixed-Integer Programs. The main idea of Stochastic Programming (SP) is to build a set of scenarios that consists of discrete realizations of uncertainties in the problem, and then optimizing the expected value of the objective criterion over all possible scenarios. In our problem, the scenarios correspond to the discrete failure states.

Combining the two technologies (simulation and optimization) exploits their complementary advantages. Discrete rate simulation is able to represent the operation of the integrated site in great detail. In fact, these types of models have been used at The Dow Chemical Company to simulate real manufacturing systems and validate them against actual operating data. Each simulation run, however, requires that design variables be fixed. Searching for an optimal design requires enumeration techniques that are time consuming and provide no guarantee of finding the optimal solution. By building and solving Mixed-Integer Linear Programming (MILP) models, one can find a guaranteed optimal solution using state-of-the-art MILP solvers such as CPLEX (ILOG, 2011). MILP models, however, are equation-oriented: the objective function and operational constraints have to be expressed in algebraic terms. In order to do so, the modeler is required to make certain simplifications of real systems. We use optimization to find a set of Pareto-optimal designs using an algebraic model of the system and simulation to evaluate in more detail the performance of these candidate designs. If the number of scenarios (discrete states) used in the optimization model is impractically large, as is the case in when there is a large number of possible failure modes, we also use the simulation model to generate a sample of the most representative scenarios. Finally, we provide some sensitivity analysis derived from the optimal solution to provide 
guidelines for fine-tuning the design using the simulation model. Figure 1 summarizes the solution process described above.

\begin{tabular}{|c|c|c|}
\hline Simulation & Optimization & Simulation \\
\hline $\begin{array}{l}\text { - Run } 5 \text { or } 10 \text { year } \\
\text { simulation of } \\
\text { superstructure to obtain } \\
\text { a sample of failure states }\end{array}$ & $\begin{array}{l}\text { - Use the sample of states } \\
\text { to build stochastic } \\
\text { programming } \\
\text { representation }\end{array}$ & $\begin{array}{l}\text {-Exploit accuracy of } \\
\text { simulation model to } \\
\text { evaluate the } \\
\text { performance of the set of } \\
\text { Pareto-optimal designs }\end{array}$ \\
\hline $\begin{array}{l}\text { - Collect statistics of the } \\
\text { states in the sample } \\
\text {-Distribution of } \\
\text { residence time } \\
\text {-Frequency } \\
\text {-Cycle Time }\end{array}$ & $\begin{array}{l}\text { - Solve the resulting MILP } \\
\text { problem to obtain a set } \\
\text { of Paretooptimal designs } \\
\text { of minimum cost and } \\
\text { maximum average } \\
\text { production rate } \\
\text { - Carry out sensitivity } \\
\text { analysis to find possible } \\
\text { search direction to fine } \\
\text { tune design }\end{array}$ & $\begin{array}{l}\text {-If necessary, finetune } \\
\text { design using sensitivity } \\
\text { analysis as a guideline to } \\
\text { search design space }\end{array}$ \\
\hline
\end{tabular}

Fig. 1 Summary of solution strategy using simulation and optimization tools

The following sections describe in more detail each of the elements in the simulation and optimization solution approach. First, we develop the MILP model of the integrated sites and explain how the resulting formulation corresponds to a two-stage Stochastic Programming (SP) problem with endogenous uncertainties. The SP formulation relies on constructing a set of failure scenarios that can be impractically large for the problem at hand. To overcome the computational complexity involved in solving large instances of the problem, we propose a novel decomposition algorithm based on Benders decomposition. Next, we describe the discrete-rate model used to simulate the integrated site. Finally, we present two case studies: an academic example and an industrial case study. The first example is meant to illustrate the results of the optimization approach. Since the number of failure modes is small, we can enumerate all discrete failure states, 
and there is no need for a simulation model to obtain a representative sample of states. We use this small example to introduce the methodology for sensitivity analysis. Since the industrial case study is significantly larger, it requires the simulation-optimizationsimulation sequence depicted in Figure 1. We use this example to explain the methodology for constructing a sample of failure states.

An important note to keep in mind is that in the SP representation, the discrete states that correspond to combinations of failure modes are called scenarios. For our purposes, the terms states and scenarios are equivalent, but the latter is used in some of the remaining sections in order to be consistent with SP literature.

\section{Mixed-Integer Linear Programming model}

The starting point for the mathematical model of the integrated site is a superstructure that corresponds to a network of processes and storage tanks. Each of the nodes in the network has the structure shown in Figure 2. The variables are described in the nomenclature section in the appendix.

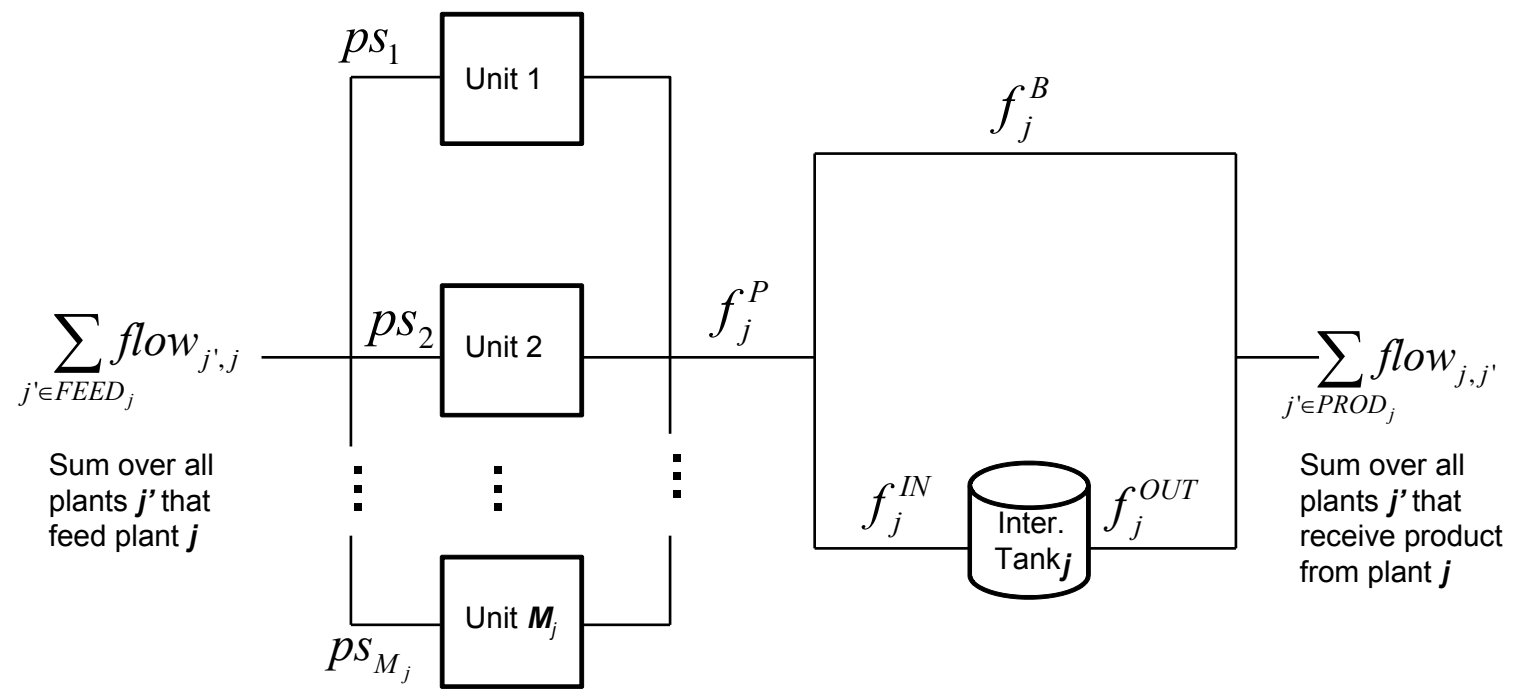

Fig. 2 Building block for plant $j$ in the integrated site

In previous work (Terrazas-Moreno et al., 2010), a mixed-integer linear programming (MILP) formulation for solving this problem was proposed in which 
exogenous uncertainties in supply and demand were also included. The most important features of the formulation are: (i) a superstructure that contains all potential parallel units and storage tanks in all plants in the process network, (ii) a state-space representation of the integrated site where each discrete state corresponds to a combination of simultaneous failure modes, and (iii) a model of intermediate storage, based on the concept of random walks (Heyman and Sobel, 1982), to determine the average and variance of the levels of material in the storage tanks as a function of the network design. The transitions between states (random process failures and repairs) follow the behavior of a continuous time Markov chain (Heyman and Sobel, 1982). This approach allows the calculation of the mean and variance of the time spent in each state, the frequency of visits to each of them, and the probabilities of finding the integrated site in any state using statistical information from historical reliability data of existing processes that resemble the ones postulated in the superstructure.

All of the above elements are integrated in an MILP formulation that maximizes the expected stochastic flexibility [E(SF)] of finished products and minimizes the capital investment required by the network design (Terrazas-Moreno et al., 2010). The bicriterion optimization problem is solved using the $\varepsilon$-constrained method (Ehrgott, 2005). The degrees of freedom are the selection of units from the superstructure, the size and location of intermediate storage tanks, and the production capacity of the plants. An important difference between our previous paper and the present one is that here we maximize average production rate instead of expected stochastic flexibility (Straub and Grossmann, 1990). The system resides during a portion of the operating horizon in states where some of the components are affected by failure modes that temporarily decrease production rate. Maximizing average production rate over a long operating time involves designing the system so that the effect of random failures is minimized and, therefore, has a similar effect on the system design as the objective of maximizing E(SF). Computation of $\mathrm{E}(\mathrm{SF})$ relies on the criterion of whether or not the system can match the demand rate in each of the discrete failure states and fails to capture the difference between a state where the production rate is slightly less than the demand rate from one where the entire system is shut down. The objective of maximizing long-term average production rate better matches industrial design criteria than the maximization of $\mathrm{E}(\mathrm{SF})$. The appendix of this 
paper contains the complete mathematical formulation. Details of the model and a description of each of the constraints can be found in Terrazas-Moreno et al. (2010).

\section{Stochastic programming representation}

We model the problem of optimal design of an integrated site (IS) as a two-stage stochastic mixed-integer linear program. The vector of first stage design decisions $d$ includes binary variables to represent the selection of production units from a superstructure and continuous variables, such as production unit capacities and storage tank sizes. Stage two decisions only involve continuous variables. A number of failure modes contained in a set $L$ can occur in the production units of the superstructure at random times. This fact introduces endogenous uncertainty to the operation of the IS. Furthermore, this uncertainty is of a discrete nature (whether or not failure $\ell \in L$ occurs) and can be modeled using a parameter $y^{\ell}$ that is 0 if failure $\ell$ occurs and 1 otherwise. The probability of a failure $\ell$ occurring at any point in time is $\operatorname{prob}^{\ell}$. We define a vector $y=\left\{y^{\ell}\right\}$ where $\ell=1, \ldots,|L|$; this vector has zeros in the positions corresponding to failures occurring simultaneously at any given time in the IS. There is a finite number of possible 0-1 combinations for the vector $y$. Each of these combinations defines a scenario in set $S$. Therefore, each scenario $s \in S$ corresponds to an instance of the vector $y$, and it can be represented as $y_{s}=\left\{y_{s}^{\ell}\right\}, \ell=1, \ldots,|L|$. Assuming independent probabilities of failures, the probability associated with each scenario is $p_{s}=\prod_{\ell: y_{s}^{\ell}=0} \operatorname{prob}^{\ell} \prod_{\ell: y_{s}^{\ell}=1}\left(1-\operatorname{prob}^{\ell}\right)$. The second stage variables $x^{s} \in \mathfrak{R}^{q}$ are used to model material flows in the integrated site (IS) for each scenario $s \in S$.

\section{Remark}

Set $S$ contains all possible failure scenarios in the superstructure, but each flowsheet selection defines a subset of relevant scenarios. The scenarios in $S$ can be aggregated - several scenarios can be projected into a single one - to derive any relevant subset for any possible flowsheet selection. 
The following mixed-integer linear programming (MILP) problem is the deterministic equivalent of the stochastic optimal design problem. It also corresponds to a compact representation of the model presented in Appendix A.

$\operatorname{Max} \sum_{s \in S} p_{s} c_{s}^{T} x_{s}-p e n^{T} s l$

s.t.

$$
\begin{array}{ll}
A_{s}^{1} x_{s}+B_{s}^{1} d \leq c^{1} & \forall s \in S \\
A_{s}^{2} x_{s} \leq \operatorname{diag}\left(B_{s}^{2} d\left(e-y_{s}\right)^{T}\right) & \forall s \in S \\
\sum_{s \in S} F_{s} x_{s}+G d-s l \leq h & \\
C_{3} d \leq \text { Capital } & \forall s \in S, s^{\prime} \in S, s^{\prime}<s,\left(s, s^{\prime}\right) \in N A \\
{\left[\begin{array}{l}
B(d)_{s, s^{\prime}} \\
x_{s}=x_{s^{\prime}}
\end{array}\right] \vee\left[\begin{array}{l}
\neg B(d)_{s, s^{\prime}} \\
x_{s} \geq 0 \\
x_{s^{\prime}} \geq 0
\end{array}\right] \quad \forall\left(s, s^{\prime}\right) \in S^{2}} & \\
d \in D, x^{s} \in \mathfrak{R}^{q}, \text { sl } \in \mathfrak{R}_{+}, \text {where } D=\left\{d \mid d_{i}=0,1, i=1, \ldots, p, d_{i} \in \mathfrak{R}_{+}^{r}, i=p+1, \ldots, r\right\} & \\
B(d)_{s, s^{\prime}}=\{\text { True, False }\} &
\end{array}
$$

The objective function (1a) represents the maximization of the average flow of product to external consumers. The coefficient $\mathrm{pen}^{T}$ penalizes the slack variables $s l$ that are introduced in constraint (1d) for guaranteeing feasibility of the subproblems and accelerating the convergence of our implementation of Benders decomposition. The penalty term is large enough to enforce the slack variable to become equal to zero at the optimal solution. In the examples we solve in this paper, we use a value of 10 for the penalty term. Constraints (1b) and (1c) represent the mass balances in the superstructure. Constraint (1c) imposes reductions on the production rates of the plants in the integrated site, according to the active failure modes in state $s$; it will have terms of the form: $p s_{m, s} \leq p c_{m}\left[1-\left(1-y_{\ell}^{s}\right)\left(r c_{\ell}\right)\right]$ (refer to constraint A11 in Appendix A). The notation $\operatorname{diag}(X)$ corresponds to the diagonal elements of the matrix $X$; and $e$ is a unitary vector. Constraint (1d) is a compact representation of the intermediate storage model we 
proposed in previous work (Terrazas-Moreno et al., 2010). Constraint (1e) corresponds to the $\varepsilon$-constraint for the bi-criterion optimization problem since it restricts the cost of a flowsheet with design variables $d$ to be less than or equal to the maximum available investment, Capital. Disjunction (D1) establishes a relationship between the operating variables of scenario $s$ and $s^{\prime}$. The Boolean variable $B(d)_{s, s^{\prime}}$ is true if scenarios $s$ and $s$ ' are indistinguishable in the flowsheet defined by $d$. It is important to notice that (D1) is not defined for every possible pair $\left(s^{\prime}, s\right)$, but only for those pairs that fulfill two conditions: a) by symmetry of the constraint set, the first condition is $s^{\prime}<s$; b) that pair $\left(s^{\prime}, s\right)$ should belong to set $N A$, which is made up of all pairs that differ in the value of exactly one element of the vector $y^{s}$. These properties are explained in detail by Goel and Grossmann (2006). We can represent (D1) using inequality constraints and a binary variable $\alpha_{s, s^{\prime}}(d)$ in (1g) and (1k) (Raman \& Grossmann, 1994), which yields the following MILP problem:

$\operatorname{Max} \sum_{s \in S} p_{s} c_{s}^{T} x_{s}-p e n^{T} s l$

s.t.

$A_{s}^{1} x_{s}+B_{s}^{1} d \leq c^{1} \quad \forall s \in S$

$A_{s}^{2} x_{s} \leq \operatorname{diag}\left(B_{s}^{2} d\left(e-y_{s}\right)^{T}\right) \quad \forall s \in S$

$\sum_{s} F_{s} x_{s}+G d-s l \leq h$

$C_{3} d \leq$ Capital

$x_{s} \leq x_{s^{\prime}}+M \alpha_{s, s^{\prime}}(d) \quad \forall s \in S, s^{\prime} \in S, s^{\prime}<s,\left(s, s^{\prime}\right) \in N A$

$x_{s} \geq x_{s^{\prime}}-M \alpha_{s, s^{\prime}}(d) \quad \forall s \in S, s^{\prime} \in S, s^{\prime}<s,\left(s, s^{\prime}\right) \in N A$

$d \in D, x^{s} \in \mathfrak{R}_{+}^{q}, s l \in \mathfrak{R}_{+}$where $D=\left\{d \mid d_{i}=0,1, i=1, \ldots, p, d_{i} \in \mathfrak{R}^{r}, i=p+1, \ldots, r\right\}$ $\alpha_{s, s^{\prime}}(d)=0,1 \quad \forall\left(s, s^{\prime}\right) \in S \times S$ 
Inequalities (1f) and (1g) are non-anticipativity constraints, where the constant $M$ is a large number that renders these inequalities to be redundant for $\alpha_{s, s^{\prime}}(d)=0$. The term $\alpha_{s, s^{\prime}}(d)$ is a function of the integrated site flowsheet and is defined as follows:

$\alpha_{s, s^{\prime}}(d)=\left\{\begin{array}{l}0 \text { if scenarios } s \text { and } s^{\prime} \text { are indistinguishable in the network topology } \\ \text { defined by d } \\ 1 \text { otherwise }\end{array}\right.$

For instance, if design $d$ does not include unit $m$ from the superstructure, then states $s, s^{\prime}$ that differ only on whether or not unit $m$ has failed are considered indistinguishable. The explicit function for $\alpha_{s, s^{\prime}}(d)$ is as follows:

$$
\alpha_{s, s^{\prime}}(d)=\sum_{m \in M l \in L_{m}} z_{m} \sigma_{1}^{s, s^{\prime}} \quad \forall j \in J, s \in S, s^{\prime} \in S, s>s^{\prime},\left(s, s^{\prime}\right) \in N A
$$

where $\sigma_{\ell}^{s, s^{\prime}}$ is a problem parameter that can be derived from the vector $y_{\ell}^{s}$. The vector $y_{\ell}^{s}$ is a fixed parameter defined in a previous section to be 1 if failure $\ell$ does not occur as part of state $s$, and 0 otherwise. Let the parameter $\sigma_{\ell}^{s, s}$ be defined as below.

$$
\sigma_{\ell}^{s, s}=\min \left\{y_{\ell}^{s}-y_{\ell}^{s^{\prime}},\left(1-y_{\ell}^{s}\right)-\left(1-y_{\ell}^{s^{\prime}}\right)\right\} \quad \forall \ell \in L, s \in S, s^{\prime} \in S, s>s^{\prime}\left(s, s^{\prime}\right) \in N A
$$

In equation (1j) $\sigma_{\ell}^{s, s^{\prime}}$ is set to one if states $s$ and $s^{\prime}$ are distinguishable with respect to failure $\boldsymbol{\ell}$; that is, if the failure occurs in one state and not in the other.

\section{Remarks}

1. The state space $S$ usually has high dimensionality, so that the number of constraints defined by $\forall s \in S, s^{\prime} \in S, s^{\prime}<s,\left(s, s^{\prime}\right) \in N A \quad$ can become computationally intractable.

2. For a fixed design $\hat{d}$, constraints (1f) and (1g) can be solved outside of the optimization problem (1). In this case, we can aggregate all indistinguishable scenarios a priori, and generate a reduced set $S_{B}$. 
3. Problem (1) with fixed $\hat{d}$ and a reduced set $S_{B}$ of scenarios corresponds to a linear programming (LP) problem. Since this LP problem excludes constraints (1f) and (1g), it results in a decrease of orders of magnitude in the number of constraints when compared against the full two-stage MILP stochastic problem.

\section{Decomposition algorithm}

\section{Basic Idea}

The algorithm we propose in this section results in a significant reduction of the number of non-anticipativity (NA) constraints that are initially considered in problem (1). This algorithm can be used (as we do in our numerical examples) with other existing modeling techniques to reduce the number of NA constraints as in Goel and Grossmann (2006). NA constraints are required only because the optimal design is a degree of freedom. The failure scenarios are built considering all units in the superstructure, but not necessarily all of these units will be part of the optimal network topology. When two or more failure scenarios are different only with respect to a failure in units that are not part of the network, a non-anticipativity constraint has to be activated to make those scenarios identical.

Figures 3 and 4 illustrate this point for a three unit example. 


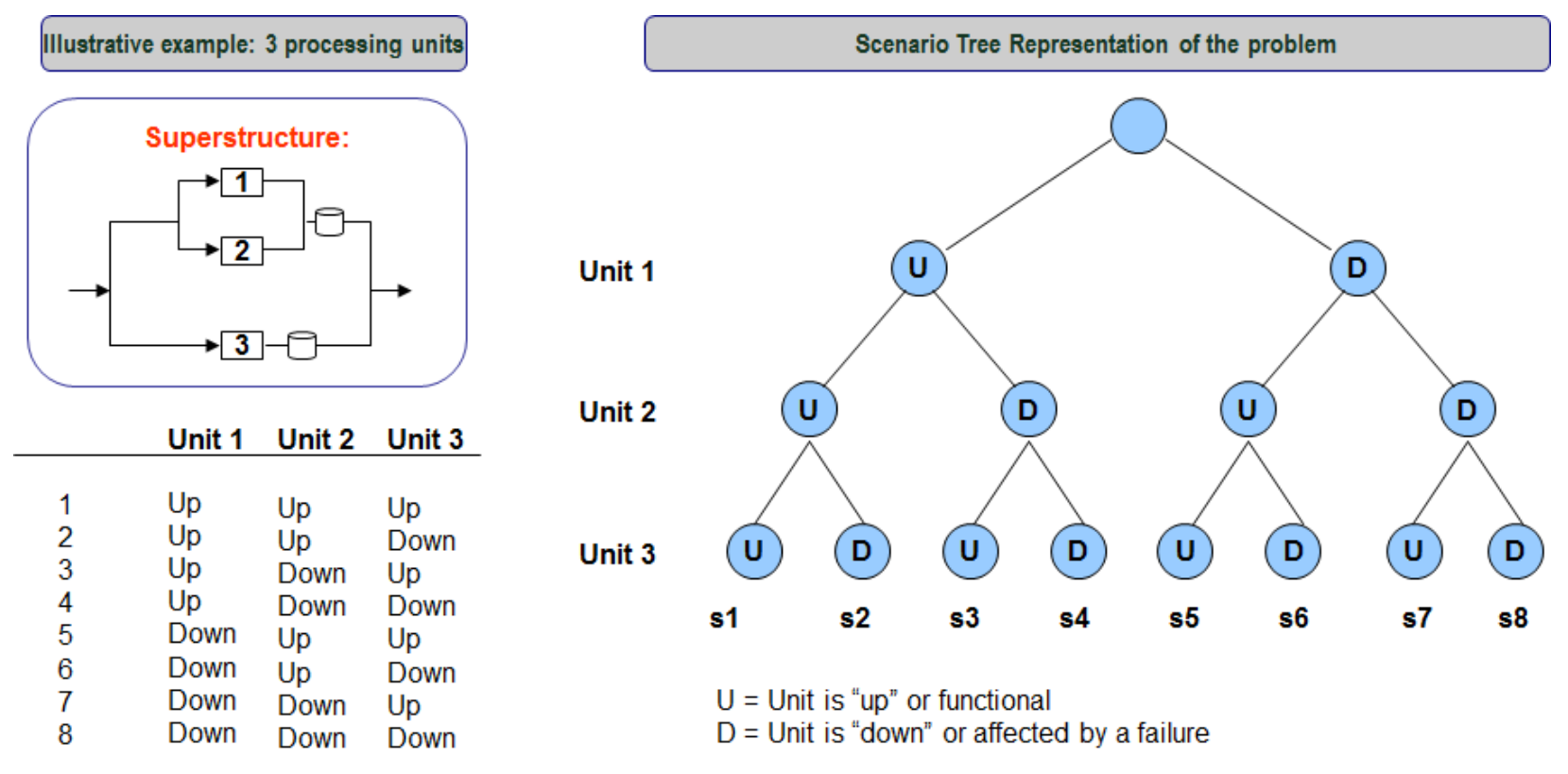

Fig. 3 Scenario tree representation of failure scenarios

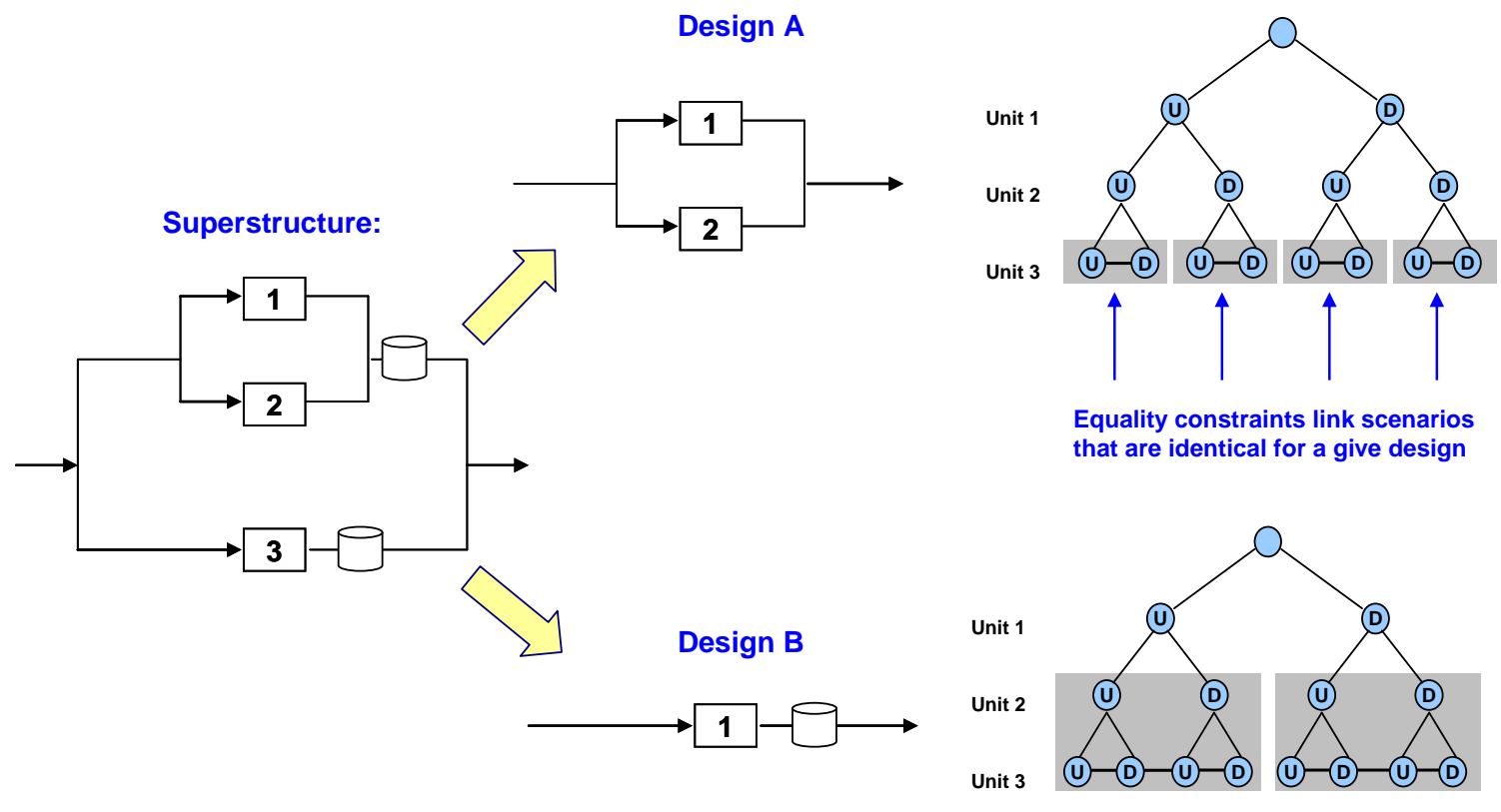

Fig. 4 NA constraints are required since flowsheet structure is a degree of freedom

The basic idea of the algorithm is to iteratively solve for the design (flowsheet) in a problem with only a few scenarios, and then solve the rest of the problem in a reduced space where only scenarios relevant to the fixed flowsheet are considered. Since only failures relevant to installed units are considered in the second step, there is no need for 
NA constraints. We use the basic concept of Benders decomposition to obtain the flowsheet in the master problem and to solve the rest of the scenarios in the subproblem. Our contribution to the method is that the subproblem is solved in a reduced space where there is a limited number of scenarios and no (or only very few) non-anticpativity constraints.

\section{Definitions}

According to what we have defined so far in the paper, we have the following sets:

$L=\{\ell: \ell$ is a failure mode in the superstructure $\}$

$S=\{s: s$ represents a combination of failure modes in $L\}$

$y^{s}=\left\{y_{\ell}^{s}: y_{\ell}^{s}=0\right.$ for active failure mode, 1 otherwise, $\left.\ell=1, \ldots,|L|\right\}$

We now include the following definitions

$\bar{L}_{k} \subseteq L$ is a subset of failures relevant to a network topology $k$

$S_{B}^{k}=\left\{s:\right.$ s represent a combination of failure modes in $\left.\bar{L}_{k}\right\}$

$S_{M}=\{s:$ subset of failure modes for master problem $\}, S_{M} \subseteq S$

$S_{M}^{C}:=$ Complement of $S_{M}$

Finally, we define a subset of the Cartesian product $S \times S_{B}^{k}$ (a function $F N_{k}: S \rightarrow S_{B}^{k}$ ) as:

$F N_{k}=\left\{\left(s^{\prime}, s\right): y_{\ell}^{s^{\prime}}=y_{\ell}^{s}, \forall \boldsymbol{\ell} \in \bar{L}_{k}\right\} \subseteq S \times S_{B}^{k}$ 
This function can be used to project the different scenarios in $S$ that are identical with respect to the failures in $L_{k}$ onto one scenario in $S_{B}^{k}$. This use of $F N_{k}$ is illustrated in Figure 5.

$$
F N_{k}=\left\{\left(s^{\prime}, s\right): y_{\ell}^{s^{\prime}}=y_{\ell}^{s}, \forall \boldsymbol{\ell} \in \bar{L}_{k}\right\} \subseteq S \times S_{B}^{k}
$$

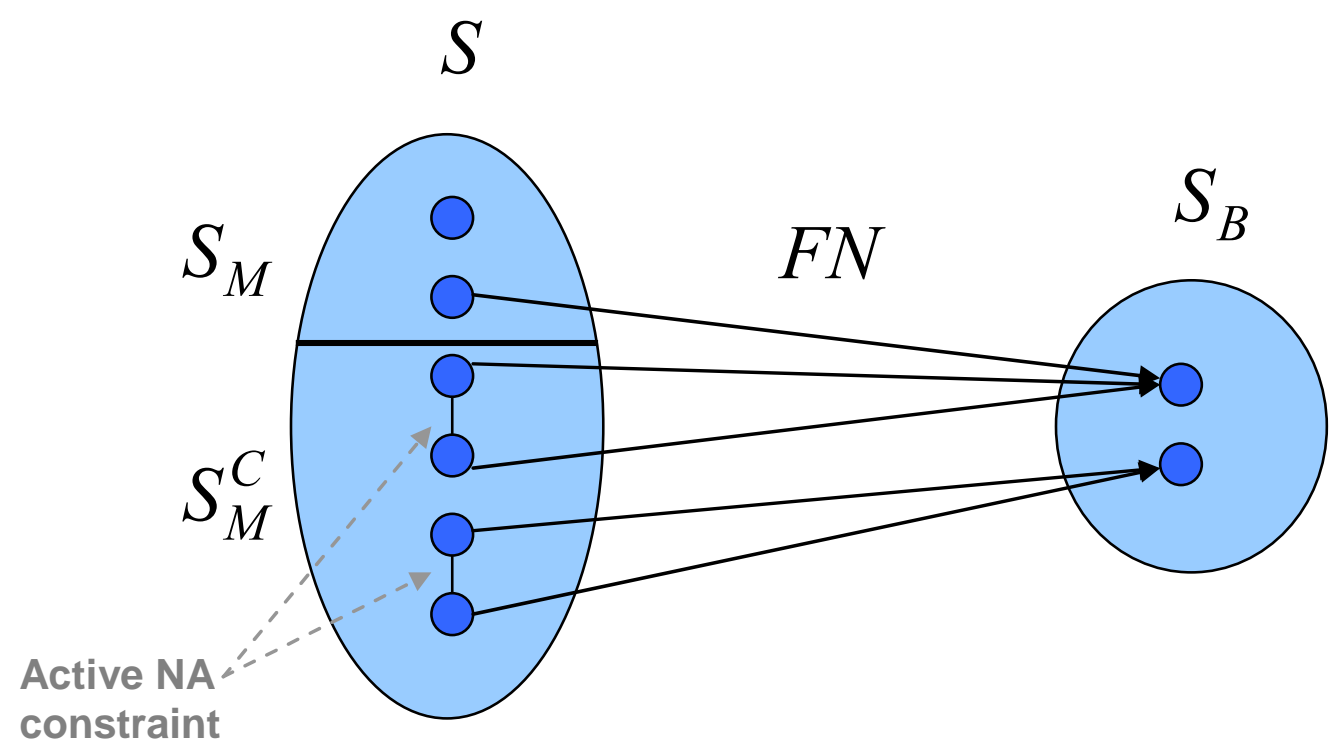

Fig. 5 Function $F N_{k}$

\section{Properties of the reduced set $S_{B}^{k}$}

In the above section, $S_{M}$ represents the failure modes considered in the master problem, while $S_{B}^{k}$ was defined as a set of scenarios that include all the relevant failure mode combinations for a fixed network topology $k$. The main idea behind the decomposition approach presented in this paper is to solve the Benders subproblem in the reduced space of $S_{B}^{k}$. For instance, let the superstructure of an integrated site have two units. Each unit has one failure mode, so $L=\left\{\boldsymbol{\ell}_{1}, \boldsymbol{\ell}_{2}\right\}$. Each vector $y_{s}=\left\{y_{s}^{\ell_{1}}, y_{s}^{\ell_{2}}\right\}$ describes a scenario $s \in S$ where $S=\{\{1,1\},\{1,0\},\{0,1\},\{0,0\}\}$. Recall that a 0 in the vector $y_{s}$ denotes the occurrence of a failure in scenario $s$. Assuming the probability of failure is small, the Benders algorithm is set up so that $S_{M}=\{\{1,1\}\}$ and its 
complement $S_{M}^{C}=\{\{1,0\},\{0,1\},\{0,0\}\}$. If in a given iteration the flowsheet obtained from the solution of the master problem includes only one unit, we would define the reduced set $\bar{L}_{k}=\left\{l_{1}\right\}$, so that $S_{B}^{k}=\{\{1\},\{0\}\}$. In this case scenario $\{1\}$ in $S_{B}^{k}$ is the projection of $\{1,0\}$ from $S$ onto $S_{B}^{k}$. Scenario $\{0\}$ is the projection of $\{0,1\},\{0,0\}$.

An important property that we require of $S_{B}^{k}$ is that the sum of the probabilities of the scenarios in the reduced set must be equal to the summation of the probabilities in the original set, i.e., $\sum_{s \in S_{B}^{k}} p_{s}^{k}=\sum_{s \in S_{M}^{C}} p_{s}$. We illustrate how to compute the probabilities $p_{s}, \forall s \in S_{B}^{k}$ in order to satisfy this condition using the example of a two-unit superstructure. There is one failure mode per unit in each of the two units, where $\operatorname{prob}^{11}=0.02$ and $\operatorname{prob}^{\mathrm{l}_{2}}=0.03$. Figure 6 shows the combinations of failure modes for scenarios in $S$ and their corresponding probabilities. It also shows the partitioning of $S$ into $S_{M}$ and $S_{M}^{C}$.

(a)

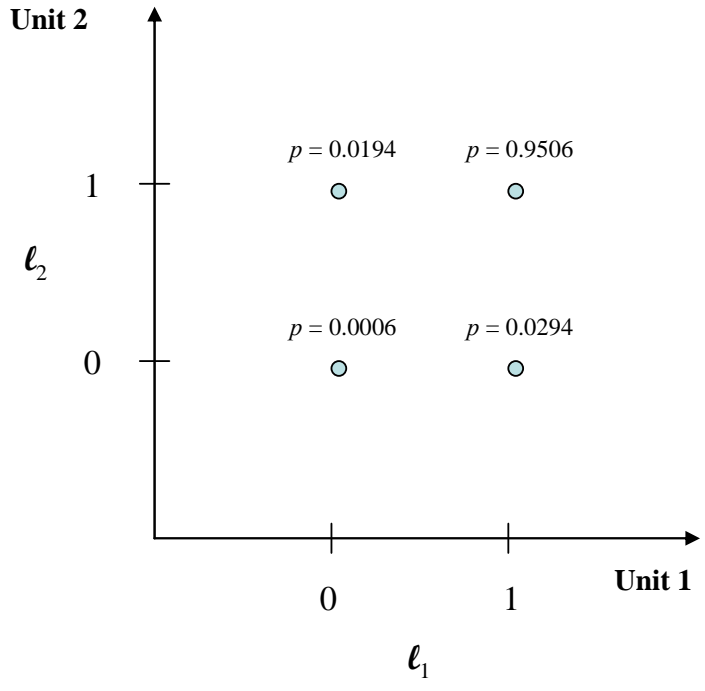

(b)

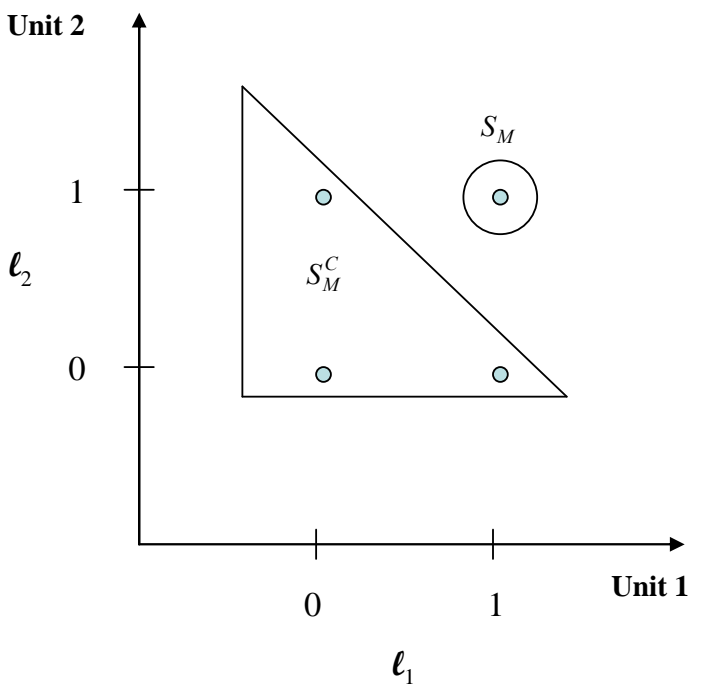

Fig. 6 Scenarios and their partitioning in illustrative example 
Once more, assume that at a given iteration of the Benders algorithm only unit one is chosen from the superstructure, and we wish to solve the subproblem in the projected space $S_{B}^{k}$. There are two states in $S_{B}^{k}$ : $\{1\}$ and $\{0\}$ corresponding to the functional and failed states of unit 1 . The first of these, $\{1\}$, is the projection of $\{1,0\}$; the second, $\{0\}$, is the projection of $\{0,1\},\{0,0\}$. The probabilities of the reduced states $\{1\}$ and $\{0\}$ are equal to the sum of the probabilities of the projected states $\{1,0\}$ and $\{0,1\},\{0,0\}$. The probability of the state $\{1\}$ is equal to the probability of $\{1,0\}$, and the probability of $\{0\}$ is the sum of the probabilities of $\{0,1\},\{0,0\}$. Figure 7 shows the reduced scenarios and their corresponding probabilities for fixed unit 1 . It can be verified that each of the probabilities in the scenarios in Figure 7 correspond to the addition of probabilities of the first and second column of Figure 6(a), considering only the states in $S_{M}^{C}$. We label the probability in the reduced space as $p_{s}^{k}$. In general, $p_{s^{\prime}}^{k}=\sum_{s \in A} p_{s}$, where $A=\left\{s: y_{s}^{l}=y_{s^{\prime}}^{l} \forall l \in \bar{L}, s \in S_{M}^{C}\right\}$.

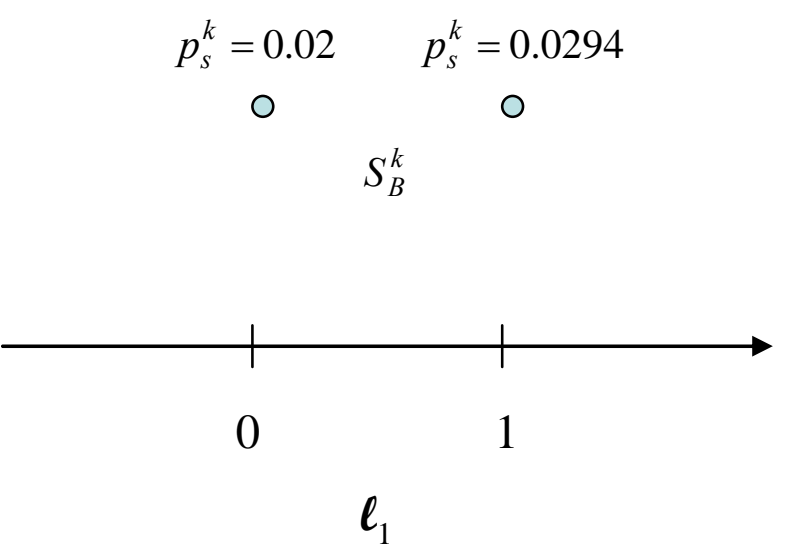

Fig. 7 Reduced scenarios in $S_{B}^{k}$ and their probabilities $p_{s}^{k}$ for fixed unit 1 


\section{Proposed algorithm}

Step 1

Define a maximum number of iterations $K_{\max }$ and the tolerance of the problem $\varepsilon$. Set the counter $K=1$, and the initial value for the lower bound $L B=-\infty$.

Select $S_{M}$ as the set of scenarios with largest probability $p_{s}$ (i.e. $p_{s} \geq \gamma$, where $\gamma$ is threshold value).

Step 2

Solve the master problem $(M)$ as defined below:

Max Constant $+\varepsilon \eta$

s.t.

$$
\begin{aligned}
\eta \leq \sum_{s \in S_{M}} p_{s} c_{s}^{T} x_{s} & +\sum_{s \in \bar{S}_{B}^{k}}\left(u_{1 s}^{k}\left(c^{1}-B_{s}^{1^{k}} d\right)+u_{2 s}^{k} \operatorname{diag}\left(B_{s}^{2^{k}} d\left(e-y_{s}\right)^{T}\right)\right)+v^{k}\left(h-G d-\sum_{s \in S_{M}} F_{s} x_{s}\right) \\
& +\sum_{s \in S_{B}^{k}} \sum_{s^{\prime}: s^{\prime} \in S_{M},\left(s, s^{\prime}\right) \in F N_{k} \cap N A} w_{s, s^{\prime}}^{k} x_{s^{\prime}}, k \neq 1, k=1, \ldots, K-1
\end{aligned}
$$

$\eta \geq L B+\varepsilon$

$\eta \leq \sum_{s \in S_{M}} p_{s} c_{s}^{T} x_{s}+\sum_{s \notin S_{M}} p_{s} c_{s}^{T} x_{s}^{U B}$

$A_{s}^{1} x_{s}+B_{s}^{1} d \leq c^{1} \quad \forall s \in S_{M}$

$A_{s}^{2} x_{s} \leq \operatorname{diag}\left(B_{s}^{2} d\left(e-y_{s}\right)^{T}\right) \quad \forall s \in S_{M}$

$\sum_{s \in S_{M}} F_{s} x_{s}+G d \leq h-\sum_{s \in S_{M}} F_{s} x_{s}^{L 0}$

$C_{3} d \leq$ Capital

$x_{s} \leq x_{s^{\prime}}+M \alpha_{s, s^{\prime}}(d) \quad \forall s \in S_{M}, s^{\prime} \in S_{M}, s^{\prime}<s,\left(s, s^{\prime}\right) \in N A$

$x_{s} \geq x_{s^{\prime}}-M \alpha_{s, s^{\prime}}(d) \quad \forall s \in S_{M}, s^{\prime} \in S_{M}, s^{\prime}<s,\left(s, s^{\prime}\right) \in N A$

$d \in D, x^{s} \in \mathfrak{R}_{+}^{q}, s l \in \mathfrak{R}_{+}$where $D=\left\{d \mid d_{i}=0,1, i=1, \ldots, p, d_{i} \in \mathfrak{R}^{r}, i=p+1, \ldots, r\right\}$

$\alpha_{s, s^{\prime}}=0,1 \quad \forall\left(s, s^{\prime}\right) \in S \times S$ 
where $u_{1 s}^{k}, u_{2 s}^{k}, v^{k}, w_{s}^{k}$ are dual variables arising in the subproblems defined in Step 4.

The master problem (M) is the bottleneck of the decomposition algorithm. To speed up the convergence of (M), we have set it up as a feasibility problem instead of a rigorous optimization problem. The value of the constant term in the objective function (M1) is of the same order of magnitude as the optimal solution to the full space problem. This value is easy to calculate since it is possible to know the productivity if no failures were present in the integrated site. Then we solve (M) using a loose tolerance in an MILP solver. The tolerance that we refer to here is the gap between the upper and lower bounds of the branch and bound method used to solve the MILP. It is not to be confused with the tolerance defined for the Benders decomposition algorithm. For instance, if the tolerance of the Benders decomposition, which is $\varepsilon$ in the nomenclature of this paper, is set to $2 \%$, we can allow a gap of $5 \%$ for the branch and bound method. In (M1) the term Constant is much larger than the term $\varepsilon \eta$, so that (M) will converge once a feasible solution is found. Although, as we have just said "the term $\varepsilon \eta$ is comparatively small", keeping it is important since it improves the quality of the feasible solution found. Equation (M2) and (M3) constrain the variable $\eta$ to be less than the dominant Benders cut but greater than a valid lower bound plus the convergence tolerance $\varepsilon$. Constraint (M4) has been added to enforce a valid upper bound on the objective function of the master problem. Note that the cardinalities of the sets of constraints (M9) and (M10) are much smaller than the cardinalities of (1f) and (1g). The solution to this problem yields the optimal values of the decision variables of the master problem at iteration $k: \hat{d}^{k}$ and $\hat{x}_{s}^{k} \forall s \in S_{M}$.

Termination criterion: Constraints (M5) - (M11) can always be trivially satisfied by not installing any unit from the superstructure and setting to zero the internal flows within the integrated site. The only possibility for (M) to be infeasible is if $\eta$ cannot satisfy constraints (M2) - (M4). This is the case only if the upper bounds for $\eta$ set by constraints (M2) and (M4) are lower than the lower bound set by constraint (M3). Therefore, the algorithm is terminated as soon as (M) is infeasible, which in turn 
guarantees that the lower bound $L B$ is within $\varepsilon$-tolerance of the optimal solution to the full space problem.

Step 3

Select the sets $\bar{L}_{k}$ and $S_{B}^{k}$ as defined above. Use the function $F N_{k}$ to compute the coefficients in the reduced space of $S_{B}^{k}$ :

$$
\begin{array}{ll}
p_{s}^{k}=\sum_{s^{\prime} s^{\prime} \in S_{M}^{C},\left(s^{\prime}, s\right) \in F_{k}} p_{s^{\prime}} & \forall s \in S_{B}^{k} \\
F_{i j_{s}}^{k}=\sum_{s^{\prime} s^{\prime} \in S_{M}^{C},\left(s^{\prime}, s\right) \in F_{k}} F_{i j_{s}}^{k} \quad & \forall s \in S_{B}^{k}, i \in\{i: \text { i rows of } F\}, j \in\{j: j \text { columns of } F\}
\end{array}
$$

The calculation of these coefficients has been explained in the previous section.

\section{Step 4}

Solve the subproblem (B) of the Benders decomposition using the reduced scenario set $S_{B}^{k}$

$\operatorname{Max} \sum_{s \in S_{B}^{k}} p_{s}^{k} c_{s}^{T} x_{s}^{k}+\sum_{s \in S_{M}} p_{s} c_{s}^{T} \hat{x}_{s}^{k}-\operatorname{pen}^{T} s l$

s.t.

$A_{s}^{1} x_{s} \leq c^{1}-B_{s}^{1} \hat{d}^{k} \quad \forall s \in S_{B}^{k}$

$A_{s}^{2} x_{s} \leq \operatorname{diag}\left(B_{s}^{2} \hat{d}^{k}\left(e-y_{s}\right)^{T}\right) \quad \forall s \in S_{B}^{k}$

$\sum_{s \in S_{B}^{k}} F_{s} x_{s}-s l \leq h-G \hat{d}^{k}-\sum_{s \in S_{M}} F_{s} \hat{x}_{s}^{k}$

$x_{s}=\hat{x}_{s^{\prime}}^{k}$

$\forall s \in S_{B}^{k}, s^{\prime} \in S_{M},\left(s, s^{\prime}\right) \in F N_{k} \cap N A$

$x^{s} \in \mathfrak{R}_{+}^{q}, s l \in \mathfrak{R}_{q}^{+}$

The non-negative slack variables $s l$ in (B4) ensure feasibility of the subproblem. A special note must be made regarding constraint (B5). The reduced set $S_{B}^{k}$ is introduced in order to eliminate the need for non-anticipativity constraints (1f) and (1g) among the scenarios in $S_{M}^{C}$, but there are still indistinguishable pairs of scenarios $\left(s, s^{\prime}\right)$ where $s$ 
belongs to $S_{M}^{C}$ and $s$ ' to $S_{M}$. The claim is that the set of constraints in (B5) is of significantly smaller cardinality than (1f) and (1g).

After solving subproblem (B), we construct the Benders cut using the objective function of the dual of (B):

$$
\begin{gathered}
\eta \leq \sum_{s \in S_{M}} p_{s} c_{s}^{T} x_{s}+\sum_{s \in \bar{S}_{B}^{k}}\left(u_{1 s}^{k}\left(e-B_{s}^{1^{k}} d\right)+u_{2 s}^{k} \operatorname{diag}\left(B_{s}^{2^{k}} d y_{s}^{T}\right)\right)+v^{k}\left(h-G d-\sum_{s \in S_{M}} F_{s} x_{s}\right) \\
+\sum_{s \in S_{B}^{k}} \sum_{s^{\prime}: s^{\prime} \in S_{M},\left(s, s^{\prime}\right) \in F N_{k} \cap N A} w_{s, s^{\prime}}^{k} x_{s^{\prime}} \quad, k \neq 1, k=1, \ldots, K-1
\end{gathered}
$$

where $u_{1 s}^{k}, u_{2 s}^{k}, v^{k}$, and $w^{k}$ are the optimal dual multipliers of constraints (B2), (B3), (B4), and (B5) at iteration $k$.

The value of the lower bound $(L B)$ is updated if the optimal value of $(\mathrm{B})$ is greater than the incumbent $L B$.

If $K=K_{\max }$, the algorithm stops. Otherwise, set $K=K+1$, and go to Step 2 .

\section{Discrete rate simulation model}

The simulation begins with the generation of discrete items, each representing a unique failure mode. Each plant in the integrated site has one or more failure modes. For each failure mode, we look up which plant is impacted by the failure. We then look up the number of parallel units for that plant and the production rate per unit. These parameters are assigned to the failure mode as attributes so the look-up is necessary only once at the beginning. Each item is unbatched to $\mathrm{N}$ items, where $\mathrm{N}=$ number of parallel units. The underlying assumption is that if multiple paralle units exist in a plant, these units fail independently of each other. For each failure mode, its time between failure (TBF) and time to repair (TTR) are calculated from distributions developed from available data. All failure modes wait for failure start, for the duration corresponding to their respective TBF. Then they wait in a queue, and each is released only when the affected unit/train is running (no ongoing failure or turnaround). When a failure mode is released from this queue, the rate and status of the affected unit is updated. All failure modes wait for repair to finish, for the duration corresponding to their respective TTR or less (only if the repair is pre-empted by a simulation logic that synchronizes certain maintenance activities). After the repair is finished for a given failure mode, the rate and status of the affected 
unit/train are restored. The TBF and TTR are recalculated, and the process is repeated. Figure 8 summarizes the simulation logic for modeling unplanned downtimes resulting from failures.

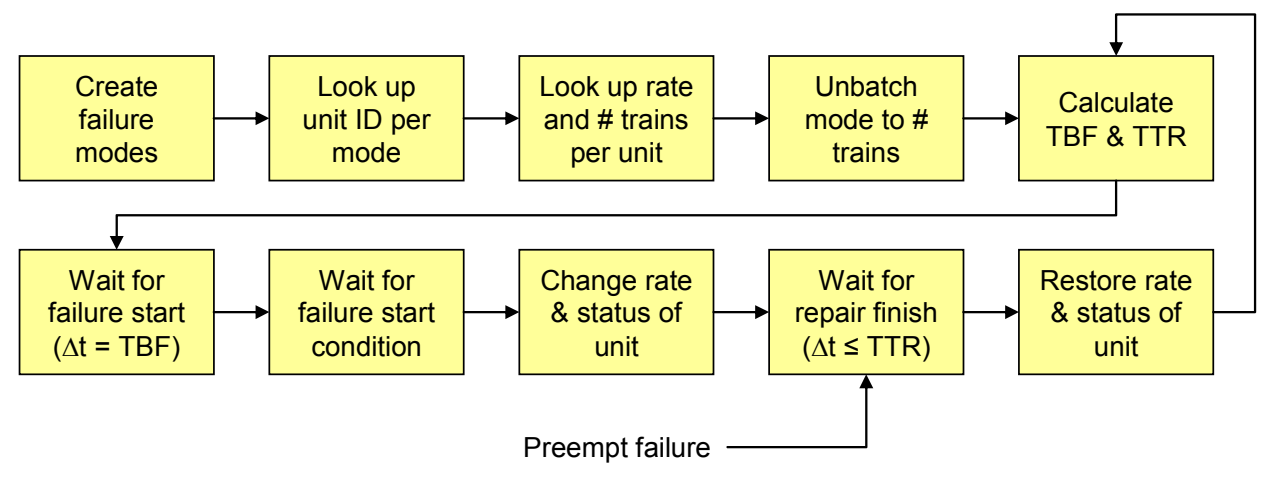

Fig. 8 Simulation logic for unplanned downtimes

A failure mode can result in the rate of zero for a complete shutdown or between zero and maximum unit capacity for rate loss. If there is an ongoing failure at a unit such that the current rate is zero, the above mentioned queue prevents any further failure modes for that unit to be activated, the assumption being that a down plant cannot fail any more. If there is an ongoing failure at a unit such that the current rate is not zero, then additional failure is possible. During the simulation, multiple failure modes can be in progress at the same time. The lowest rate of all failure modes in progress is chosen as the rate of a particular unit. The existence of more than one parallel unit per plant is accounted for in the updating of this rate input, depending on the status of all units. For example, if one unit is up and the other is down, then the rate input would be half the plant capacity.

\section{Application to the design of integrated sites (IS)}

\section{Illustrative Example}

We use the small example shown in Figure 9 that was presented in our previous work (Terrazas-Moreno et al., 2010) to illustrate the proposed algorithm. The model and process data required to solve this example are available in Appendix A. We solve the full space version of the problem and then decompose it using the proposed algorithm for 
different values of capital investment in order to obtain a set of Pareto-optimal solutions. All results were obtained using the MILP solver CPLEX version 12.1, running on GAMS 23.3, with a $2.8 \mathrm{GHz}$ Intel Pentium 4 processor and 2.5 GB RAM.

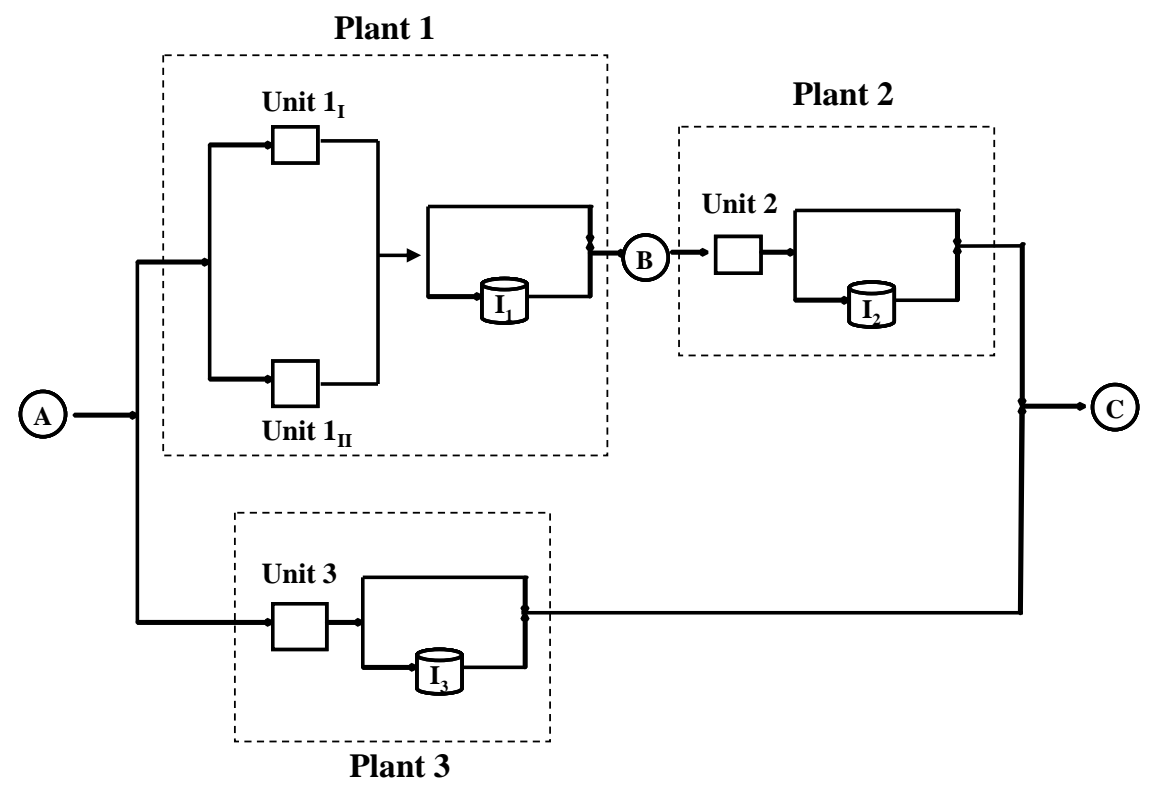

Fig. 9 Integrated Site for the production of C from A

The Pareto-optimal solutions are shown in Figure 10. Two specific network structures are shown for the Pareto-optimal points A and B in Figure 11.

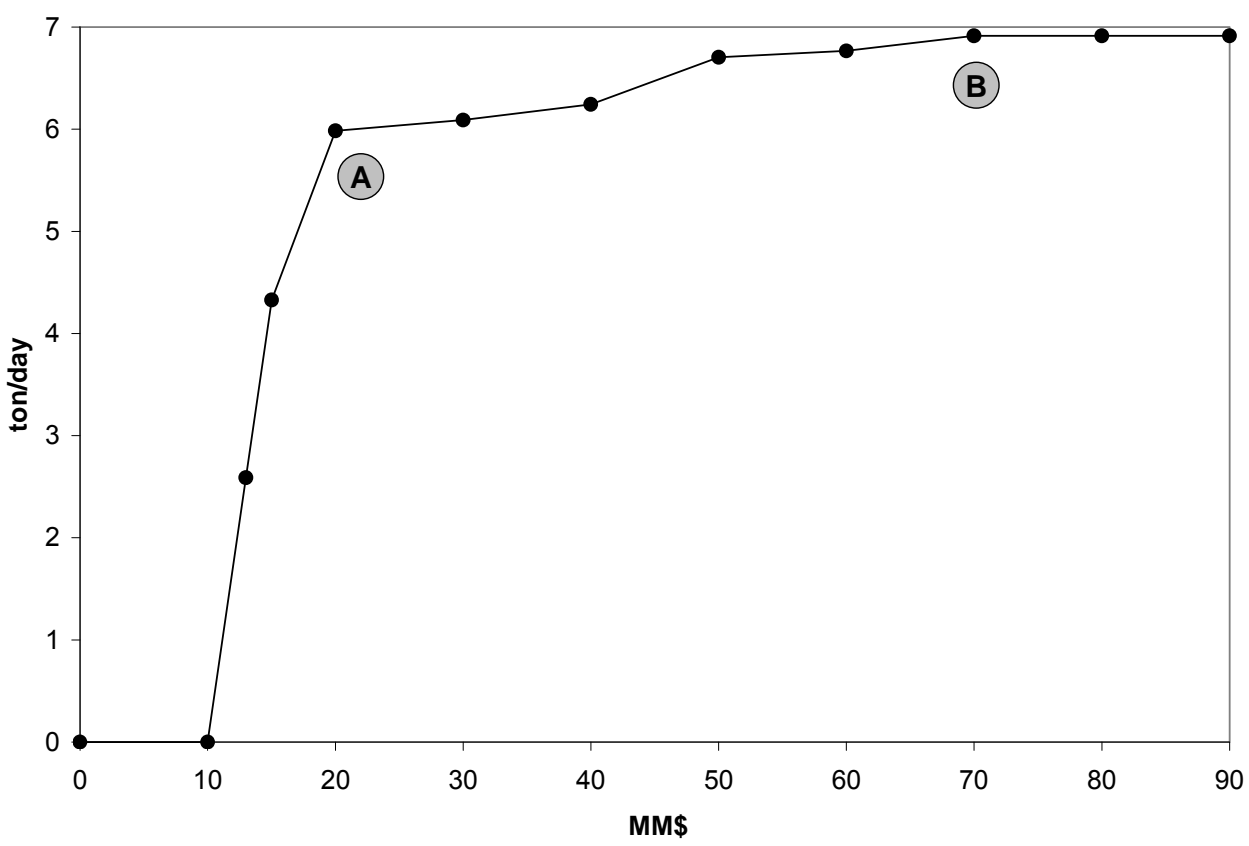

Fig. 10 Pareto-set of optimal solutions for Example 1 


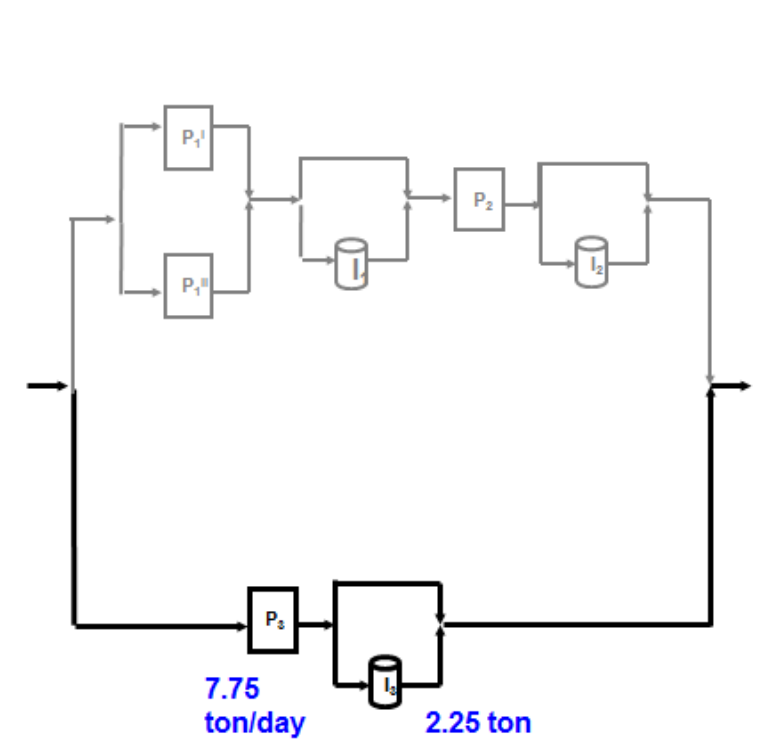

\section{Capital Investment $\quad=20$ MM USD Average production rate $=6.00$ ton/day}

(a) Configuration $\mathrm{A}$

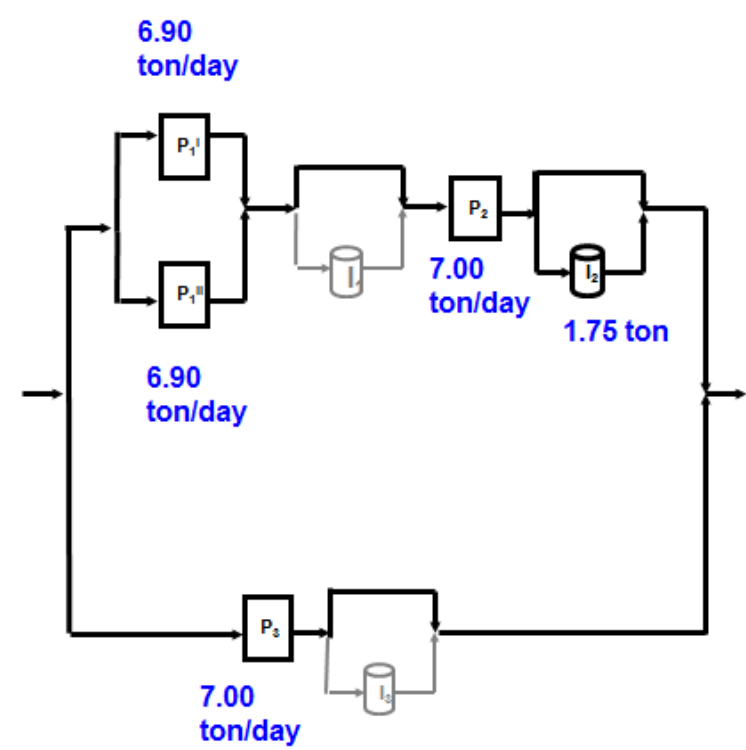

Capital Investment $\quad=70 \mathrm{MM}$ USD Average production rate $=6.92$ ton $/$ day

(b) Configuration B

Fig. 11 Network configurations for two Pareto-optimal solutions

The network in Figure 11(a) is designed to operate relying on process 3 and a large storage tank. In fact, 2.25 tons is the upper bound we set for the volume of the storage tank after plant 3. The network configuration that corresponds to point B in Figure 10 belongs to a section of the optimal Pareto set where only marginal improvements in average production rate are achieved at the expense of large additional investment. Thus, the large spare capacities, the redundant units, and the relatively large storage tank in Figure 11(b).

Tables 1 and 2 contain the problem sizes and the results that correspond to the full space model and to the decomposition strategy. Figure 12 shows the convergence of the decomposition algorithm in 14 iterations for one value of capital investment (Capital $=$ $\$ 60 \mathrm{MM})$. The lower bound before iteration 4 is a large negative number as a result of the slack variable in the objective function and equation (B4) having a large value. Without the slack variable the subproblem would have been infeasible. It is interesting to notice that, even for this small example made up of 4 processing units, the number of constraints in the full space model is significantly larger than the sum of the constraints in the master problem and subproblem (see Table 1). This is a consequence of the reduction in the 
number of non-anticipativity constraints in (1f) and (1g), and in the number of scenarios in the subproblem. Table 2 shows the results obtained by the full space and Benders decomposition methods for different values of capacity investments. The example is so small that each Pareto-optimal solution can be obtained in a fraction of a second of CPU time either using the decomposition algorithm or directly solving the full space model.

Table 1 Statistics of illustrative example

\begin{tabular}{|c|c|c|c|}
\hline & $\begin{array}{c}\text { Discrete } \\
\text { variables }\end{array}$ & $\begin{array}{c}\text { Cont. } \\
\text { variables }\end{array}$ & $\begin{array}{l}\text { Const } \\
\text { raints }\end{array}$ \\
\hline Full space & 4 & 1557 & 1670 \\
\hline Master Problem* & 4 & 502 & 370 \\
\hline Subproblem ${ }^{\S}$ & 0 & 265 & 177 \\
\hline
\end{tabular}

Table 2 Results of illustrative example

\begin{tabular}{ccc}
\hline $\begin{array}{c}\text { Capital Investment } \\
\text { MM USD }\end{array}$ & \multicolumn{2}{c}{ Optimal Solution [ton/day] } \\
Full space & $\begin{array}{c}\text { Sub problem } \\
\text { (2\% tolerance) }\end{array}$ \\
\hline 13 & 2.61 & 2.59 \\
15 & 4.35 & 4.33 \\
20 & 6.10 & 5.99 \\
30 & 6.10 & 6.09 \\
40 & 6.25 & 6.25 \\
50 & 6.76 & 6.71 \\
60 & 6.77 & 6.77 \\
70 & 6.92 & 6.92 \\
80 & 6.92 & 6.92 \\
90 & 6.92 & 6.92 \\
\hline
\end{tabular}




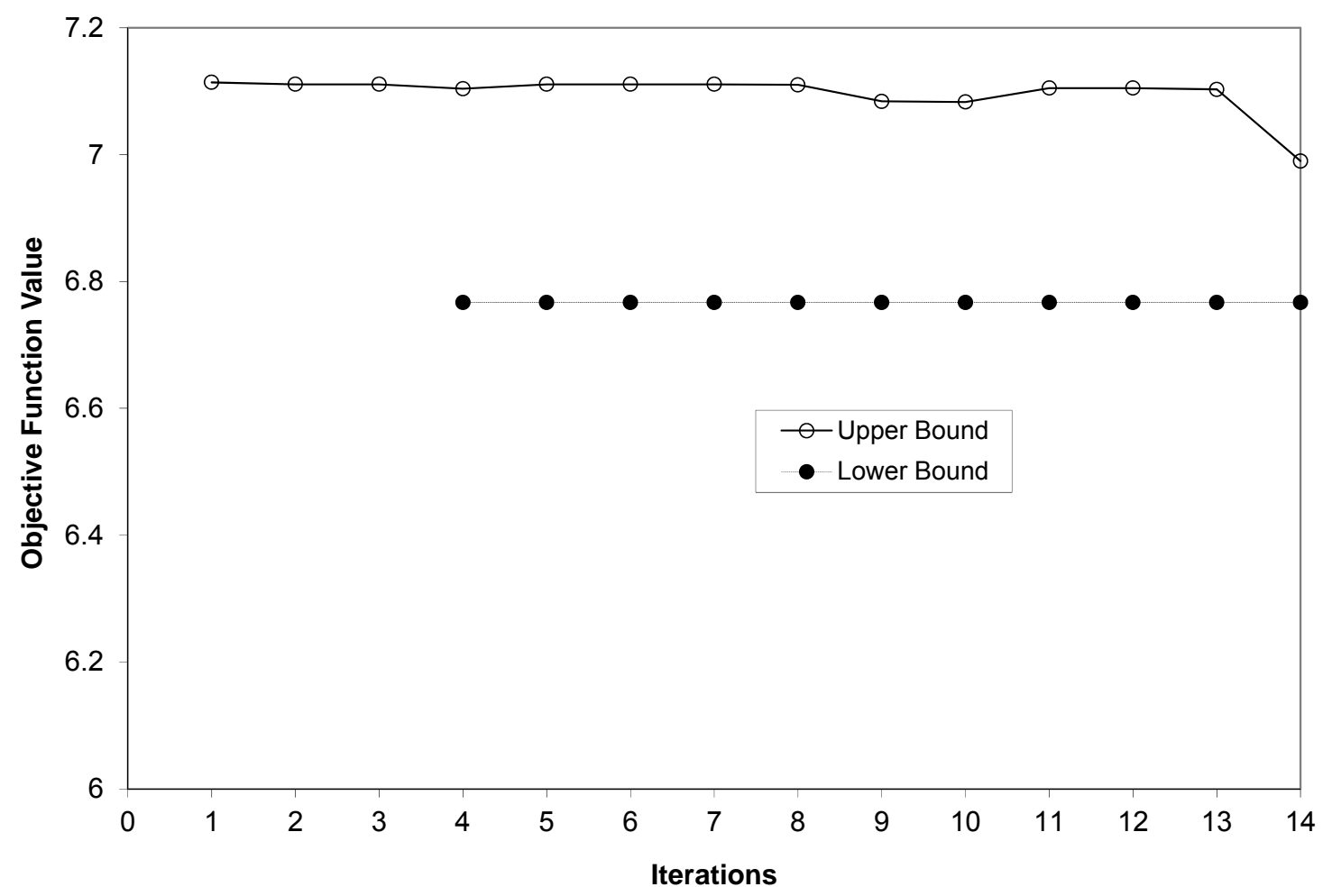

Fig. 12 Iterations of master problem and sub problem in the illustrative example for $\$ 60 \mathrm{MM}$ of investment

\section{Sensitivity Analysis}

The Pareto-optimal solution that corresponds to point A in Figure 10 represents an inflection point in the Pareto-optimal front. Due to the fact that further marginal increments in average production rate require large sums of extra capital investment, an industrial design team would be interested in understanding the factors that limit the performance of this design. For example, it could be more cost effective trying to improve the reliability characteristics of key components in the design, rather than adding parallel production trains or increasing the capacity of existing ones. It could also be the case that small changes in the volume of storage tanks or capacity of production units can yield significant improvements in average production rate. This information can be very useful with the optimization-simulation approach proposed in this paper. These teams could use it to search efficiently the design space around one or more Pareto-optimal solutions with discrete-rate simulation. The sensitivity analysis described below has the 
objective of providing this type of information to such a design team, so that it can be used as a guideline to fine-tune the design in a cost-effective way.

The first step to carry out the sensitivity analysis is to fix the selection of units in the superstructure to that in the Pareto-optimal point of interest. We then construct the set of scenarios relevant to this selection in the same manner as when setting up the subproblem in the decomposition algorithm. Using this collection of scenarios as set $S$, and with a fixed selection of units (fixed $z_{m}$ ), we solve the full space problem described in Appendix A (equations A1 - A22) with the following two additional constraints.

$$
\begin{array}{ll}
p c_{m}^{*} \leq p c_{m} \leq p c_{m}^{*} & \forall m \in M \\
v_{j, n}^{*} \leq v_{j, n} \leq v_{j, n}^{*} & \forall j \in J, n \in N
\end{array}
$$

where $p c_{m}^{*}$ and $v_{j, n}^{*}$ are the capacity of unit $m$ and the volume of the tank for product $n$ after plant $j$ in the Pareto-optimal design being analyzed. Any solver for LP problems, such as CPLEX, provides the reduced costs at the optimal solution. The reduced costs, which are the dual variables at the active bounds, correspond to the derivatives of the objective function with respect to perturbations on the right-hand sides of each of the constraints in the optimization formulation (Chvatal, 1983). By reading reduced costs of constraints (S1) and (S2), we obtain the values of the following derivatives:

$$
\frac{\partial \text { (Average Production Rate) }}{\partial\left(p c_{m}^{*}\right)} \quad \forall m \in M
$$

and

$$
\frac{\partial(\text { Average Production Rate })}{\partial\left(v_{j, n}^{*}\right)} \quad \forall j \in J, n \in N
$$

These derivatives show the sensitivity of the optimal solution for marginal increments in the design variables. In this way, the relative magnitudes of the sensitivities can be used as guidelines for fine-tuning the design using simulation tools.

Next, we are interested in finding the derivatives of the objective function with respect to the probabilities of each failure mode, that is, $\frac{\partial(\text { Average Production Rate) }}{\partial\left(p_{\ell}\right)} \quad \forall \ell \in L$. 
This information can be used to determine key failure modes and look for ways to improve the reliability characteristics of the corresponding components in the design. The probabilities of being in an operational state with respect to failure $\ell, p_{\ell}$, do not appear explicitly in the MILP model defined by equations (A1) - (A22). However, the probability of each failures scenario (discrete state) , $p r o b^{s}$, which is a function of the probabilities of independent failure modes, does appear in constraints (A1), (A12) and (A13). Note that we assume that $v r t^{s} f r^{s}=p r o b^{s}$ in constraint (A13), which is true when failures follow exponential distributions but an approximation in any other case. Knowing this, we carry out the following computations where $A P R$ stands for average production rate:

$$
\frac{\partial(A P R)}{\partial\left(p_{\ell}\right)}=\sum_{s \in S} \frac{\partial(A P R)}{\partial\left(\operatorname{prob}^{s}\right)} \frac{\partial\left(\mathrm{prob}^{s}\right)}{\partial\left(p_{\ell}\right)},
$$

where

$$
\frac{\partial(A P R)}{\partial\left(p^{\prime} r b^{s}\right)}=\sum_{j \in J} \sum_{n \in N}\left[\frac{\partial(A P R)}{\partial\left(A 12_{j, n}\right)} \frac{\partial\left(A 12_{j, n}\right)}{\partial\left(\operatorname{prob}^{s}\right)}+\frac{\partial(A P R)}{\partial\left(A 13_{j, n}\right)} \frac{\partial\left(A 13_{j, n}\right)}{\partial\left(p r o b^{s}\right)}\right]+\frac{\partial(A 1)}{\partial\left(\operatorname{prob}^{s}\right)}
$$

and

$$
\frac{\partial\left(p r o b^{s}\right)}{\partial\left(p_{\ell}\right)}=\prod_{\ell:\left\{y_{\ell}^{s}=1\right\} \ell^{\prime} \neq \ell} p_{\ell^{\prime}} \prod_{\ell^{\prime}:\left\{y_{\ell}^{s}=0\right\}}\left(1-p_{\ell^{\prime}}\right) \text { if } y_{\ell}^{s}=1
$$

or

$$
\frac{\partial\left(p r o b^{s}\right)}{\partial\left(p_{\ell}\right)}=\prod_{\ell:\left\{y_{\ell}^{s}=1\right\}} p_{\ell^{\prime}} \prod_{\ell:\left\{y_{\ell}^{s}=0\right\}, \ell^{\prime} \neq \ell}\left(1-p_{\ell^{\prime}}\right) \text { if } y_{\ell}^{s}=0
$$

In equation (S7), $A 12$ and $A 13$ stand for the right-hand sides of equations (A12) and (A13) in Appendix A, and $A 1$ corresponds to the objective function. The values $\frac{\partial(A P R)}{\partial\left(A 12_{j, n}\right)}$ and $\frac{\partial(A P R)}{\partial\left(A 13_{j, n}\right)}$ are the dual variables of the corresponding constraints, and they are obtained from the output of an LP solver. The partial derivatives $\frac{\partial\left(A 12_{j, n}\right)}{\partial\left(\operatorname{prob}^{s}\right)}$, $\frac{\partial\left(A 13_{j, n}\right)}{\partial\left(\text { prob }^{s}\right)}$ and $\frac{\partial(A 1)}{\partial\left(\text { prob }^{s}\right)}$ are obtained analytically from constraints (A12) and (A13) and 
the objective function (A1). Finally, equations (S8) and (S9) are the result of differentiating the function $p r o b^{s}=\prod_{\ell:\left\{y_{\ell}^{s}=1\right\}} p_{\ell^{\prime}} \prod_{\ell:\left\{y_{\ell}^{s}=0\right\}}\left(1-p_{\ell^{\prime}}\right)$ that determines the probability of each failure state as a combination of the probabilities of the independent failure modes.

Table 3 contains the results of the sensitivity analysis of the design labeled as $\mathrm{A}$ in Figure 10. Note: design A only involves plant 3.

Table 3 Sensitivity analysis of design corresponding to $\$ 20 \mathrm{MM} *$

\begin{tabular}{|c|c|}
\hline$\frac{\partial(A P R)}{\partial\left(v_{3, C}^{*}\right)}$ & $\sim 0$ \\
\hline$\frac{\partial(A P R)}{\partial\left(p c_{3}^{*}\right)}$ & $\sim 0$ \\
\hline$\frac{\partial(A P R)}{\partial\left(p_{3}\right)}$ & 6.98 \\
\hline
\end{tabular}

*This design only includes unit 3 from the superstructure

The results in Table 3 indicate that marginal changes in the design variables around their optimal values have little effect on the objective function. In fact, this is consistent with the small slope after point A in Figure 10. In contrast, increasing the probability of being in an operational state of plant 3 has a large potential impact on the average production rate. To give the value of the derivative $\frac{\partial(A P R)}{\partial\left(p_{3}\right)}$ a more tangible meaning, we can calculate the effect of a 5\% increase in the ratio of MTBF over MTTR for plant 3. After some algebraic manipulation of the expression $p_{3}=\frac{M T B F_{3}}{M T B F_{3}+M T T R_{3}}$ we get: $\triangle A P R(5 \%$ increase in MTTF $/ M T T R)=\left[\frac{1.05}{1+0.05 p_{\ell}}-1\right] \frac{\partial(A P R)}{\partial\left(p_{3}\right)}=0.044$

Thus, an increase of $5 \%$ on the mentioned availability could result in the design corresponding to \$20 MM going from 6.095 mass-unit/hr to 6.139 mass-unit/hr. This 
option would be preferred over increasing the capital investment from \$20 MM to over \$30 MM.

\section{Large-scale Example}

This section describes the computational results of the proposed algorithm for solving an industrial-sized process network. Figure 13 shows the 9 processing plants that constitute this network. Each of the plants in this integrated site represents the production of a chemical that can be shipped to external markets or used as raw material in a downstream process. In the latter case, the integrated site could be part of a very large chemical production site made up of several "smaller" integrated sites. In this example we use a concave cost function (the common six-tenths rule (Biegler et al., 1997)) for capturing the effect of different processing capacities on the capital investment required by processing units. We use a piece-wise linear approximation in order to keep the linearity of the model.

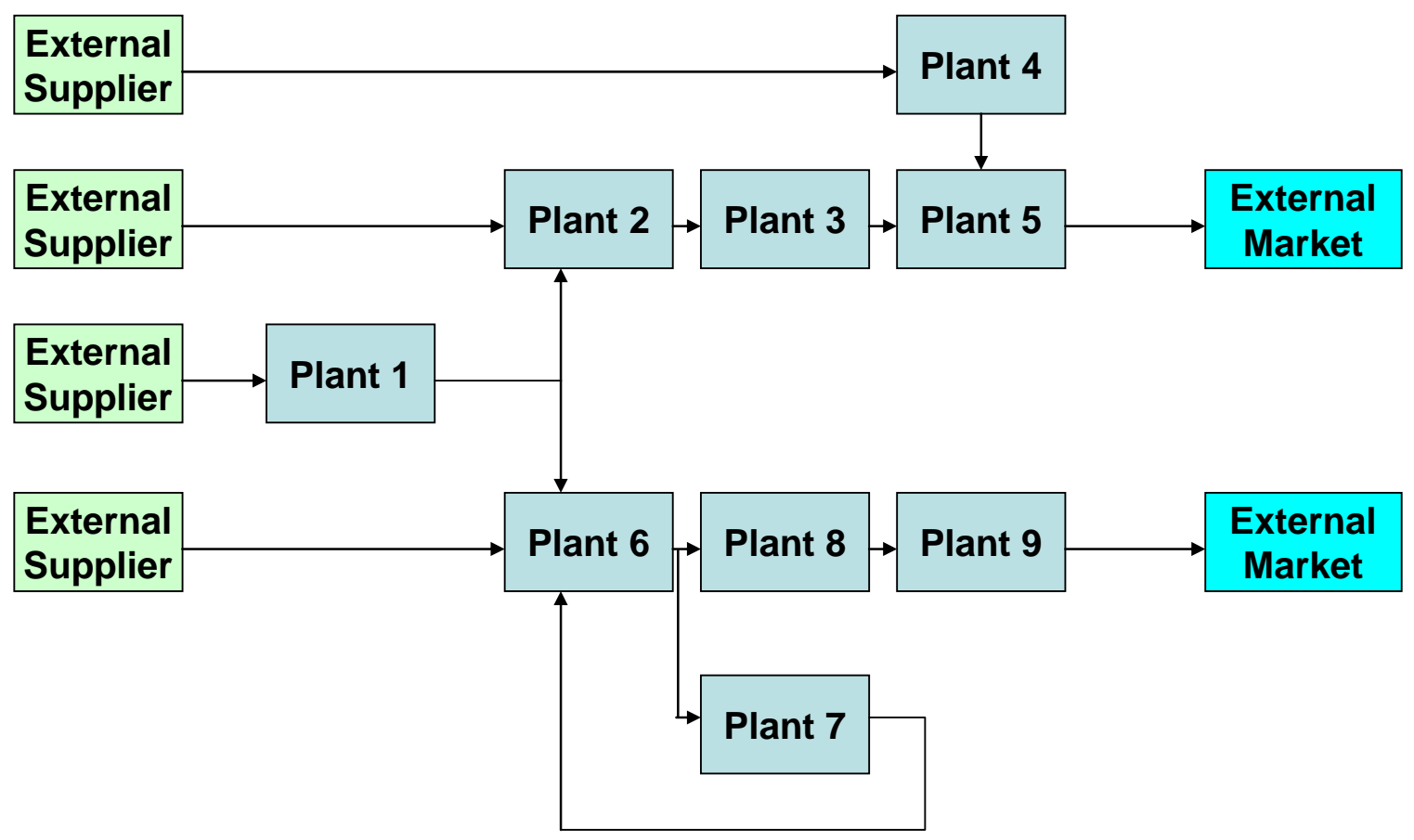

Fig. 13 Industrial integrated site in large-scale example

Each of the 9 plants that constitute the integrated site is modeled as a continuous process with one or several inputs and one output. We postulate a superstructure with two parallel 
production units in each plant and a storage tank after each plant except after plants 5 and 9, where the corresponding product cannot be stored. Each unit in the superstructure is subjected to different partial (decreased capacity) and total random failure modes. The distribution of the failure times is exponential, while the repair times follow a normal distribution. The total number of failures in the superstructure is 198.

Solving this industrial case study with the formulation in Appendix A using the decomposition framework presents two main challenges. One of them is the dimensionality: the resulting space for the units in the superstructure consists of $2^{198}$ discrete states (scenarios). Even with the decomposition algorithm, we are not able to handle this problem size. The second challenge is that the normal distribution of the repair times causes the variance of the residence time in each state to be different than that calculated assuming an exponential distribution. As indicated in our previous work (Terrazas-Moreno et al., 2010), the asymptotic values of the probabilities of the states and the mean residence time can be obtained analytically for any type of distribution as long as we have the mean failure and repair times. Unfortunately, this is not the case for the variance of the residence times. We overcome these two challenges by building a discrete event simulation model that we can use to collect a sample of scenarios. This technique is basically a Monte Carlo sampling procedure.

Sampling of scenarios and validation of designs using the discrete event simulation model

One use of the simulation model is to build a sample of scenarios for our problem. As the simulation runs, it generates a list of items (failures) and their attributes (time between failures and time to repair). It also registers a generation time for each of the items (failures). Scenarios (states) in the problem at hand are described by a set of failures occurring simultaneously in the integrated site. From the list of items mentioned above, we can construct a list of scenarios or states and their durations. Appendix B contains the details of the methodology to construct the list of scenarios from the list of failure items created in discrete event simulation. Since the scenarios are generated randomly, we can consider the method as a Monte Carlo sampling. The number of scenarios is finite, and a few of them are highly more likely than the rest, so we can 
expect many repetitions of the same scenario as we sample. The sampling method allows us to calculate probabilities as well as mean and variance of residence time in each scenario. The size of the sample can be determined by the desired level of accuracy in the solution to our two-stage stochastic programming problem. The variance estimator of the solution to a stochastic programming problem is given by Shapiro (2000):

$$
S(n)=\sqrt{\frac{\sum_{s=1}^{n}\left(E[o b j]-o b j_{s}\right)^{2}}{n-1}}
$$

where $n$ is the number of scenarios and $o b j$ is the objective value. Let $z_{\alpha / 2}$ be the normal standard deviate (corresponding to a normal distribution with zero-mean and unitary standard deviation) for a confidence interval of $1-\alpha$. In other words, $\operatorname{Pr}\left(z \leq z_{\alpha / 2}\right)=1-\alpha / 2$, for $z \sim N(0,1)$. For a confidence interval of 95\%, $z_{\alpha / 2}=1.96$. To obtain the exact number of scenarios we follow the steps in You et al. (2009):

1) Obtain a sample for one year of operation to approximate $S(n)$.

2) Define $z_{\alpha / 2}$ and a desired interval $H$ so that the solution to the stochastic programming problem is within an interval of $\left[o b j_{\text {sample }}-H, o b j_{\text {sample }}+H\right]$ with confidence of $1-\alpha$.

3) Determine the size of the sample as:

$$
N=\left[\frac{z_{\alpha / 2} S(n)}{H}\right]^{2}
$$

For $H=2.5$ mass/hour (recall that the objective function is the expected throughput of the integrated site) and a confidence level of $95 \%$ we need a sample size of 2878 scenarios. We found that by simulating 10 years of plant operation we can construct this sample. Intuitively, one would expect that excluding scenarios that are not relevant after 10 years of plant has a minimal effect on the optimal design of the integrated site. 


\section{Numerical result}

We simulated 10 year of operation of the integrated site and obtained a sample of 2973 scenarios. The statistics corresponding to the 10 most frequently encountered scenarios are shown in Table 4.

Table 4 Statistics of most probable failure scenarios

\begin{tabular}{|c|c|c|c|c|}
\hline Scenario & Probability & $\begin{array}{c}\text { Mean residence } \\
\text { time }[\mathrm{hr}]\end{array}$ & $\begin{array}{c}\text { Frequency of visits } \\
{\left[1 /\left(\mathrm{hr} \times 10^{3}\right)\right]}\end{array}$ & $\begin{array}{c}\text { Plants in failure } \\
\text { mode }\end{array}$ \\
\hline 1 & 0.231 & 28 & 8.0 & None \\
\hline 2 & 0.022 & 23 & 0.9 & Plant 8 \\
\hline 3 & 0.020 & 20 & 1.0 & Plant 8 \\
\hline 4 & 0.020 & 25 & 0.8 & Plant 8 \\
\hline 5 & 0.019 & 27 & 0.7 & Plant 2 \\
\hline 6 & 0.013 & 18 & 0.7 & Plant 2 \\
\hline 7 & 0.012 & 21 & 0.6 & Plant 5 \\
\hline 8 & 0.010 & 25 & 0.4 & Pant 9 \\
\hline 9 & 0.009 & 21 & 0.4 & Plant 8 \\
\hline 10 & 0.008 & 28 & 0.3 & Plant 9 \\
\hline
\end{tabular}

Table 4 contains some valuable information. For instance, from the first row we know that the integrated site will operate without any active failure mode around $23 \%$ of the time and that a failure will occur approximately every 28 hours. Another important piece of information is that 4 out of the remaining 9 most common failure scenarios are due to some failure mode in Plant 8.

After obtaining the sample of failure scenarios, we attempted to solve the problem in full space of 2973 scenarios (without decomposition) and using the proposed decomposition algorithm in a workstation with a $2.40 \mathrm{GHz}$ Intel Core2 Quad CPU processor and 8 GB RAM. The results were obtained with the MILP solver CPLEX 12.1.0 running in GAMS 23.3

The set of Pareto-optimal solutions in Figure 14 was obtained first by solving the full space model which required 31 hours. In contrast, using the decomposition algorithm with a $2 \%$ convergence tolerance, the Pareto curve can be obtained in 9 hours. Table 5 shows the comparison in terms of CPU times for both approaches. An important note is 
that the solution times in the table do not include the time GAMS required to generate the model, which was nearly as long as the solution time for the full space model and not more than a few minutes with the decomposition algorithm. Table 6 shows the problem sizes of the decomposed and full space formulations. Finally, Table 7 shows the details of three of the Pareto optimal network configurations in Figure 14.

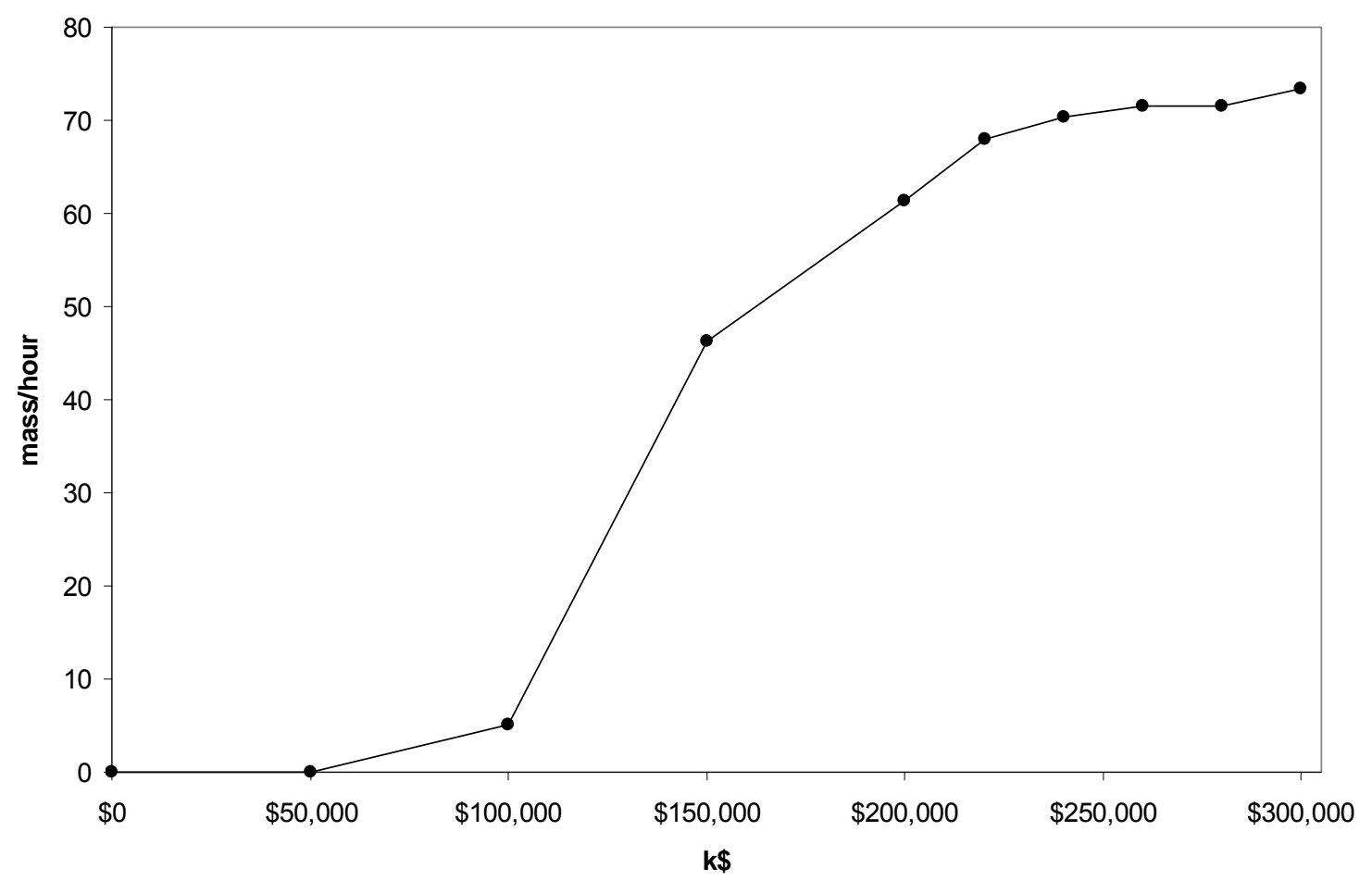

Fig. 14 Pareto-optimal solutions for Example 2 using a random sample of 2973 scenarios 
Table 5 Performances of decomposition algorithm and full space solution using sample of scenarios

\begin{tabular}{|c|c|c|c|c|c|}
\hline \multirow[b]{2}{*}{$\begin{array}{c}\text { Capital Investment } \\
k \text { U SD }\end{array}$} & \multicolumn{2}{|c|}{ Full space } & \multicolumn{3}{|c|}{ Decomposition algorithm } \\
\hline & $\begin{array}{l}\text { Solution } \\
\text { mass } / h r\end{array}$ & $\begin{array}{l}\text { CPU time } \\
\text { sec }\end{array}$ & $\begin{array}{l}\text { Upper Bound } \\
\text { mass/hr }\end{array}$ & $\begin{array}{l}\text { Lower Bound } \\
\text { mass } / h r\end{array}$ & $\begin{array}{l}\text { CPU time } \\
\text { sec }\end{array}$ \\
\hline$\$ 100,000$ & 5.20 & 1,052 & 5.26 & 5.16 & 1,324 \\
\hline$\$ 150,000$ & 46.83 & 4,980 & 47.28 & 46.35 & 6,431 \\
\hline$\$ 200,000$ & 62.12 & 15,376 & 62.52 & 61.29 & 3,817 \\
\hline$\$ 220,000$ & 68.30 & 23,271 & 69.27 & 67.91 & 3,813 \\
\hline$\$ 240,000$ & 70.77 & 12,645 & 71.66 & 70.26 & 4,277 \\
\hline$\$ 260,000$ & 72.31 & 21,611 & 72.97 & 71.54 & 3,308 \\
\hline$\$ 280,000$ & 73.27 & 18,082 & 72.95 & 71.52 & 5,109 \\
\hline \multirow[t]{2}{*}{$\$ 300,000$} & 73.97 & 15,245 & 74.92 & 73.46 & 3,080 \\
\hline & Total & 31.18 & hrs & Total & 8.66 \\
\hline
\end{tabular}

Table 6 Problem size corresponding to industrial case study

\begin{tabular}{cccc}
\hline & $\begin{array}{c}\text { Discrete } \\
\text { variables }\end{array}$ & $\begin{array}{c}\text { Cont. } \\
\text { variables }\end{array}$ & Constraints \\
\hline Full space & 144 & $4,753,154$ & $1,864,942$ \\
Master Problem* & 144 & 599,136 & 203,574 \\
Subproblem & 0 & $4,183,206$ & 877,194
\end{tabular}

* Master problem at iteration 1 
Table 7 Optimal Designs for different capital investments using a random sample of scenarios

\begin{tabular}{|c|c|c|c|c|c|c|}
\hline & \multicolumn{2}{|c|}{$\begin{array}{l}200 \text { MM USD } \\
\text { Average Production Rate } \\
61.29 \text { mass } / \mathrm{hr}\end{array}$} & & \multicolumn{2}{|c|}{$\begin{array}{l}220 \text { MM USD } \\
\text { Average Production Rate } \\
67.91 \mathrm{mass} / \mathrm{hr}\end{array}$} & \multirow[b]{2}{*}{$\begin{array}{l}\text { Storage } \\
\text { size } \\
\text { [ton] }\end{array}$} \\
\hline Plant & $\begin{array}{l}\text { Production } \\
\text { Units }\end{array}$ & $\begin{array}{l}\text { Capacity } \\
\text { per unit } \\
\text { [ton/hr] }\end{array}$ & $\begin{array}{l}\text { Storage } \\
\text { size } \\
\text { [ton] }\end{array}$ & $\begin{array}{l}\text { Production } \\
\text { Units }\end{array}$ & $\begin{array}{l}\text { Capacity } \\
\text { per unit } \\
\text { [ton/hr] }\end{array}$ & \\
\hline 1 & 1 & 74.8 & - & 1 & 88.2 & - \\
\hline 2 & 1 & 77.1 & - & 1 & 87.8 & - \\
\hline 3 & 1 & 58.7 & - & 1 & 64.4 & 3750 \\
\hline 4 & 1 & 37.5 & - & 1 & 42.8 & - \\
\hline 5 & 1 & 121.7 & - & 1 & 126.4 & - \\
\hline 6 & 1 & 105.23 & - & 1 & 154.1 & - \\
\hline 7 & 1 & 86.0 & - & 1 & 117.6 & 446 \\
\hline 8 & 1 & 28.4 & - & 1 & 42.4 & 9 \\
\hline \multirow[t]{2}{*}{9} & 1 & 44.1 & - & 1 & 67.6 & - \\
\hline & \multicolumn{3}{|c|}{$\begin{array}{l}\text { Average Production Rate } \\
71.62 \mathrm{mass} / \mathrm{hr}\end{array}$} & & & \\
\hline Plant & $\begin{array}{l}\text { Production } \\
\text { Units }\end{array}$ & $\begin{array}{l}\text { Capacity } \\
\text { per unit } \\
\text { [ton/hr] }\end{array}$ & $\begin{array}{l}\text { Storage } \\
\text { size } \\
\text { [ton] }\end{array}$ & & & \\
\hline 1 & 2 & 73.9 & - & & & \\
\hline 2 & 1 & 96.9 & - & & & \\
\hline 3 & 1 & 76.3 & 3750.0 & & & \\
\hline 4 & 1 & 42.8 & 3210.0 & & & \\
\hline 5 & 1 & 135.91 & - & & & \\
\hline 6 & 1 & 137.7 & - & & & \\
\hline 7 & 1 & 111.0 & 7770.0 & & & \\
\hline 8 & 1 & 42.4 & 93 & & & \\
\hline 9 & 2 & 52.0 & - & & & \\
\hline
\end{tabular}




\section{Sensitivity analysis}

We carry out a sensitivity analysis around the design corresponding to \$220 MM, following the same procedure outlined in Example 1. The analysis predicts that there is no marginal benefit in increasing the size of the storage tanks and that the design finetuning should focus on small increases on the capacity of plants 1 and 9. The sensitivity analysis with respect to probabilities in failure modes revealed that improving by $5 \%$ the ratio of MTBF over MTTR of one of the failure modes in either plant 2 or plant 8 could increase the average production rate from $68 \mathrm{ton} / \mathrm{hr}$ to close to $70 \mathrm{ton} / \mathrm{hr}$. This is consistent with the data in Table 4 where it can be seen that the most frequent failure modes occur in Plant 8 and Plant 2.

\section{Simulation of the Pareto-optimal designs}

We use the same simulation model previously used for obtaining the sample of failure modes to evaluate the performance of the Pareto-optimal designs from the MILP optimization model. As we have indicated before, the simulation model is able to reproduce the real system to a greater extent than the optimization model, given the simplifications that have to be assumed to develop the MILP model. Therefore, simulating the design obtained in the optimization step allows us to observe the average production rate (objective function of the optimization model) of the integrated site under more realistic assumptions than those incorporated in the constraints of the MILP model. Since we perform three simulations per configuration, we report average and standard deviation for each metric. Figure 15 shows the Pareto-optimal curve obtained through optimization and the corresponding performance fixing the network configuration and capacities in simulation runs. The error bars above and below the points that correspond to simulation are set to one standard deviation obtained when running each point three times. As can be seen, the agreement between the optimization and simulation models is very good. 


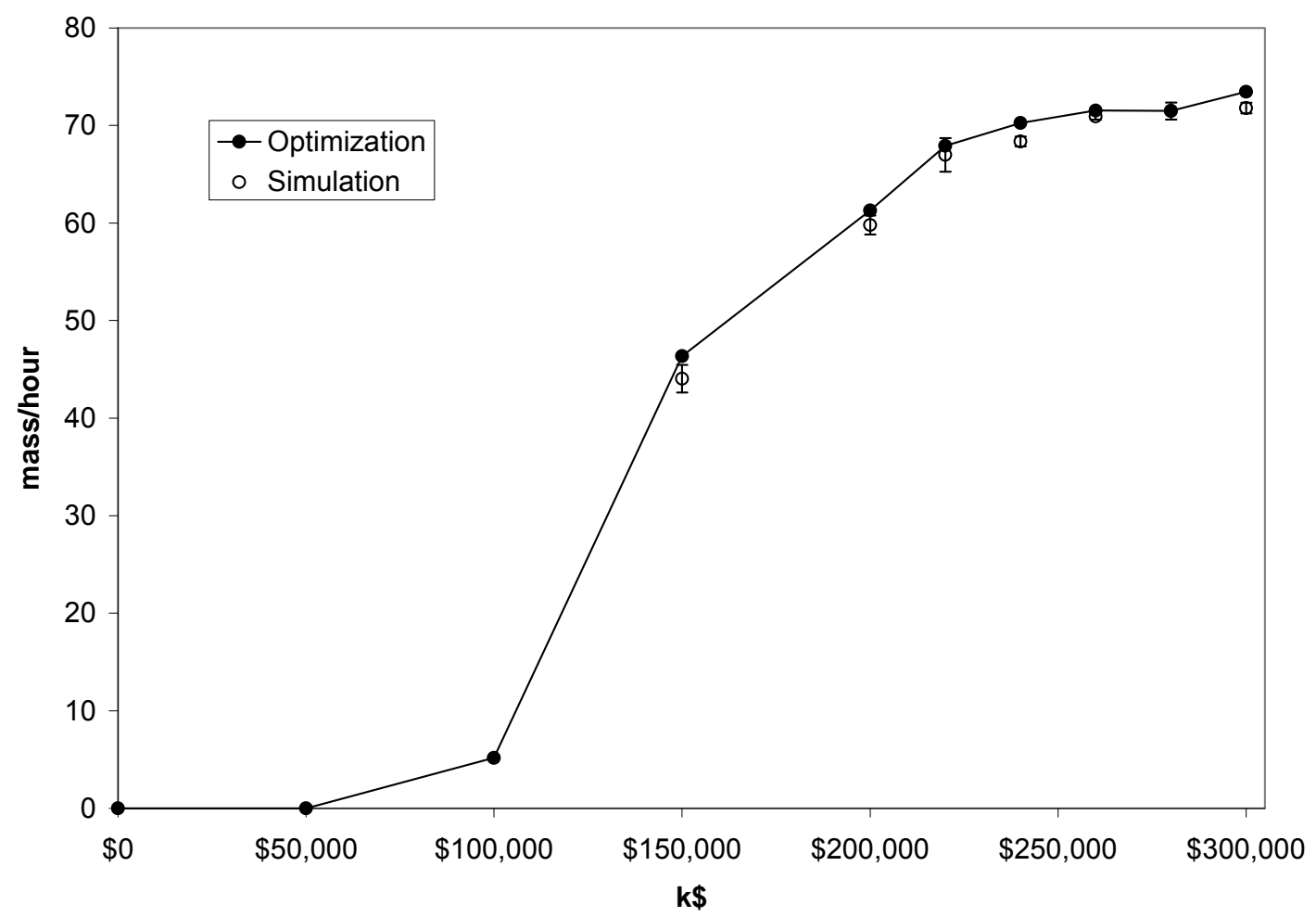

Fig. 15 Comparison of simulation results vs. optimization results for some Pareto-optimal designs

\section{Conclusions}

In this paper, we have addressed the problem of integrated site design under uncertainty as a two-stage stochastic programming with endogenous uncertainties. In order to overcome the exponential growth in the number of scenarios required for modeling uncertainty, we proposed a decomposition algorithm based on Benders decomposition. Our main contribution is to exploit the problem structure in a way that allows the solution of Benders subproblems in a reduced space. The result is that the number of scenarios required is much smaller than with the full space problem. One of the main advantages of the proposed method is that the decomposed model requires significantly fewer nonanticipativity constraints.

The solution approach was tested in two case studies, one of them being an industrial processing network in which Monte Carlo Sampling was used to reduce the number of states. In the second case study, the decomposition algorithm reduces the solution time for the complete Pareto-set from 31 to 9 hours. The integration with discrete rate simulation for validating/refining results increases the likelihood that the 
algorithmic method presented here will be accepted as a computational tool in an industrial setting.

\section{Acknowledgment}

We acknowledge Jee H. Park from the Process Optimization group at The Dow Chemical Company for his contributions during project discussions, and The Dow Chemical Company for the financial support for this work. We also acknowledge the support of ImagineThat, Inc. through the academic software grant.

\section{References}

Acevedo J. and E. N. Pistikopoulos (1998). Stochastic optimization based algorithms for process synthesis under uncertainty. Computers and Chemical Engineering, 22, 647 671.

BASF (2010). $\quad$ http://www.basf.com/group/corporate/en/investor-relations/basf brief/verbund/ludwigshafen-site. Accessed on May 6th, 2010.

Benders J. F. (1962). Partitioning procedures for solving mixed variables programming problems. Numersiche Mathematik, 4, 238 - 252.

Biegler L. T., I. E. Grossmann and A. W. Westerberg, Systematic Methods of Chemical Process Design. Prentice Hall, New Jersey (1997).

Billinton R. and R. N. Allan, Reliability Evaluation of Engineering Systems. Plenum Press, New York (1992).

Chvatal C., Linear Programming. W. H. Freeman and Company, New York (1983).

Davies K. M. and C. L. E. Swartz, (2008). MILP Formulations for Optimal Steady-State Buffer Level and Flexible Maintenance Scheduling. MASc Thesis, McMaster University.

Dow (2007) . http://news.dow.com/dow_news/corporate/2007/20070712a.htm. Accessed on May 6th 2010.

Geoffrion A. M. (1972).Generalized Benders Decomposition. JOTA, 10, 237 - 260.

Goel V. and I. E. Grossmann (2006). A Class of stochastic programs with decision dependent uncertainty. Math. Program. (Ser. B), 108, 355 - 394. 
Heyman D. P. and M. J. Sobel, Stochastic Models in Operations Research, Volume I. McGraw-Hill, New York (1982).

Ierapetritou M. G., J. Acevedo, and E. N. Pistikopoulos (1996). An optimization approach for process engineering problems under uncertainty. Computers and Chemical Engineering, 20, 703 - 709.

ILOG (2011) $\quad$ http://www-01.ibm.com/software/integration/optimization/cplexoptimizer/ Accessed on January 15th 2011.

Imagine That Inc. (2007). ExtendSim User Guide, Release 7. San Jose, CA 95119

Imagine That Inc. (2010) http://www.extendsim.com/index.html Accessed on March 23, 2011

Jonsbraten T. W., R. J. B. Wets and D. L. Woodruff (1998). Annals of Operations Research, 82, $83-106$.

Liu C., Y. Fan and F. Ordonez (2009). A two-stage stochastic programming model for transportation network protection. Computers and Operations Research, 36(5), 1582 -1590 .

Miller S., J. Owens and D. Deans (2006). From requirements development to verification - Designing reliability into a large scale chemical manufacturing system. Reliability and Maintainability Symposium, 2006. RAM ’06 Annual, 641 - 648.

Pistikopoulos E. N. (1995).Uncertainty in process design and operations. Computers and Chemical Engineering, 19, S553 - S563.

Pistikopoulos E. N., T. V. Thomaidis, A. Melin, and M.G. Ierapetritou (1996). Flexibility, reliability and maintenance considerations in batch plant design under uncertainty. Computers and Chemical Engineering, 20, S1209 - S1214.

Pistikopoulos E. N., C. G. Vassiliados, J. Arvela and L.G. Papageorgiou, (2001). Interaction of maintenance and production planning for multipurpose process plants a system effectiveness approach. Industrial and Engineering Chemistry Research, 40, $3195-3207$.

Raman R. and I. E. Grossmann (1994). Modeling and computational techniques for logic based integer programming. Computers and Chemical Engineering, 18, 563 - 578.

Santoso T., S. Ahmed, M. Goetschalckx and A. Shapiro (2005). A stochastic programming approach for supply chain network design under uncertainty. European Journal of Operational Research, 167, 96 - 115. 
Straub D. A. and I. E. Grossmann (1990). Integrated stochastic metric of flexibility for systems with discrete state and continuous parameter uncertainties. Computers and Chemical Engineering, 14(9), 967 - 985.

Straub D. A. and I. E. Grossmann (1993). Design Optimization of Stochastic Flexibility. Computers and Chemical Engineering, 17(4), 339 - 354.

Terrazas-Moreno S., I. E. Grossmann, J. M. Wassick and S. J. Bury (2010). Optimal design of reliable integrated chemical production sites. Computers and Chemical Engineering, 34(12), 1919 - 1936.

Van Slyke R. and R. J. B. Wets (1969). L-shaped linear programs with applications to optimal control and stochastic programming. SIAM Journal on Applied Mathematics, $17,638-663$.

Wassick, J. M. (2009). Enterprise-wide optimization in an integrated chemical complex. Computers and Chemical Engineering, 33(12), 1950 - 1963. 


\section{Appendix A}

MILP stochastic programming model for optimal design at reliable integrated sites

Sets/Index

$J / j$

$S / s$

$N / n$

$M / m$

$L / \ell$

FEED

$\operatorname{PROD}_{j}$

$I N P U T_{j}$

OUTPUT $_{j}$

$R A W$

FINISHED

$M_{j}$

$L_{m}$

$N A$

Variables

flow $_{j, j^{\prime}, n, s}$
Plant or large-scale chemical processes

Discrete states or scenarios

Products

Production units within the plants

Failure modes

Subset of $J$ that contains plants that supply material to $j$

Subset of $J$ that contains plants that consume products from

j

Subset of $N$ that contains inputs for $j$

Subset of $N$ that contains outputs of $j$

Subset of $\mathrm{N}$ that contains raw materials obtained from an external supplier

Subset of $\mathrm{N}$ that contains finished products delivered to external consumers

Subset of $M$ that contains the units that belong to plant $j$

Subset of $L$ that contains the failure modes that correspond

to unit $m$

tuples of states $\left(s, s^{\prime}\right)$ that differ in only one element of the vector $y^{\ell}$. This set is used to define non-anticipativity constraints

flow of material $n$ from plant $j$ to plant $j$ ' during state/scenario $s$ 
$f_{j, n, s}^{P}$

$f_{j, n, s}^{B}$

$f_{j, n, s}^{I N}$

$f_{j, n, s}^{O U T}$

$p s_{m, s}$

$\delta_{j, n}^{s}$

$\overline{i n v}_{j, n}$

$s \tilde{d}_{j, n}$

$v_{j, n}$

$p c_{m}$

$z_{m}$

$\alpha_{s, s^{\prime}}(d)$

Parameters

$y_{s}^{\ell}$ flow of material $n$ out of processing units in plant $j$ during state/scenario $s$

flow of material $n$ that bypasses the storage tank in plant $j$ during state/scenario $s$

flow of material $n$ that goes into the storage tank in plant $j$ during state/scenario $s$

flow of material $n$ that comes out of the storage tank in plant $j$ during state/scenario $s$

processing stream out of unit $m$ during state/scenario $s$ accumulation (positive) or depletion (negative) rate for storage of product $n$ in plant $j$

average inventory level in storage tank of product $n$ in plant j

standard deviation of inventory level in storage tank of product $n$ in plant $j$

volume of intermediate storage tank for product $n$ in plant $j$ processing capacity of unit $m$

1 if unit $m$ is selected from the superstructure, 0 otherwise

1 if states $s$ and $s$ ' are different considering the network topology defined by $d, 0$ if they are indistinguishable

0 if failure mode $\boldsymbol{\ell}$ occurs when the integrated site is state $s$, 1 otherwise 


$\begin{array}{ll}r c_{\ell} & \text { fraction of maximum production rate lost as a result of } \\ & \text { failure mode } \ell \\ \alpha_{m} & \text { mass balance coefficient (output to input ratio) of unit } m \\ R M_{n} & \text { Maximum rate of supply of external raw material } n \\ D F_{n} & \text { Maximum rate of external demand of finished product } n \\ \tau_{j, n}^{+} & \text {Number of standard deviations added to the average } \\ & \text { inventory level of product } n \text { in intermediate tank after plant } \\ \tau_{j, n}^{-} & j \\ & \text { Number of standard deviations subtracted from the average } \\ & \text { inventory level of product } n \text { in intermediate tank after plant } \\ & j \\ v_{j, n}^{U P} & \text { Maximum size of storage tank after plant } j \text { for product } n \\ p r o b^{s} & \text { Probability of finding state/scenario } s \text { in stochastic steady } \\ & \text { state } \\ & \text { mean residence time in state/scenario } s \text { in stochastic steady } \\ & \text { state } \\ \sigma_{\ell}^{s, s} & \text { variance of the distribution of residence time in } \\ & \text { state/scenario } s \text { in stochastic steady state } \\ & \text { frequency of visits to state/scenario } s \text { in stochastic steady } \\ & \text { state } \\ & 1 \text { if failure } \ell \text { occurs in state } s \text { or } s \text { ' but not in both, } 0 \text { if } \ell \\ & \text { occurs in both or in none of the states } \\ & \end{array}$

Mathematical model

$\max$ Average rate $=\sum_{s=1}^{S} \operatorname{prob}^{s} \sum_{j^{\prime} \in F E E D_{j}}\left[\sum_{n \in O U T P U T_{j^{\prime}} \cap I N P U T_{j} \cap F I N I S H E D}\right.$ flow $\left._{j^{\prime}, j, n, s}\right]$ 


$$
\begin{aligned}
& \sum_{m \in M} P W L_{m}(p c)+\sum_{j \in J} \sum_{n \in N} P W L_{v}(v)+\sum_{m \in M} \beta_{m}^{f i x} z_{m} \leq \varepsilon \\
& \sum_{j^{\prime} \in F E E D_{j}}\left[\sum_{n \in O U T P U T_{j} \cap \cap I N P U T_{j}} \text { flow }_{j, j, s}\right]=\sum_{m \in M_{j}} p s_{m, s} \quad \forall j \in J, s \in S \\
& \frac{\sum_{j^{\prime} \in F E E D_{j}} \text { flow }_{j^{\prime}, j, n, s}}{\sum_{j^{\prime} \in F E E D_{j}} \text { flow }_{j^{\prime}, j, \bar{n}, s}}=\vartheta_{j, n}^{\bar{n}} \quad \forall j \in J, n \in N, \bar{n} \in \operatorname{Nref}_{j}, s \in S \\
& \sum_{m \in M_{j}} \alpha_{m} p s_{m, s}=f_{j, n, s}^{P} \quad \forall j \in J, n \in \text { OUTPUT }_{j}, s \in S \\
& f_{j, n, s}^{P}=f_{j, n, s}^{I N}+f_{j, n, s}^{B} \quad \forall j \in J, n \in \text { OUTPUT }_{j}, s \in S \\
& \sum_{j^{\prime} \in \text { PROD }_{j}} \text { flow }_{j, j^{\prime}, n, s}=f_{j, n}^{\text {OUT }}+f_{j, n, s}^{B} \quad \forall j \in J, n \in \text { OUTPUT }_{j}, s \in S \\
& \text { flow }_{j^{\prime}, j, n, s}=0 \quad \forall j^{\prime} \in J, j \in J, n \notin \text { OUTPUT }_{j^{\prime}}, s \in S \\
& \sum_{j \in P R O D_{\text {supplier }}} \text { flow }_{\text {supplier }, j, n, s} \leq R M_{n} \quad \forall n \in \text { RAW, } s \in S \\
& \sum_{j \in F E E D_{\text {consumer }}} \text { flow }_{j, \text { consumer }, n, s} \leq D F_{n} \quad \forall n \in \text { FINISHED, } s \in S \\
& p s_{m, s} \leq p c_{m}\left[1-\left(1-y_{\ell}^{s}\right)\left(r c_{\ell}\right)\right] \quad \forall m \in M, \ell \in L_{m}, s \in S \\
& \sum_{s \in S} \delta_{j, n}^{s} \text { prob }^{s}=0 \quad \forall j \in J, n \in N \\
& s \tilde{d}_{j, n}=\sum_{s \in S}\left|\delta_{j, n}^{s}\right| \sqrt{v r t^{s} f r^{s} t} \quad \forall j \in J, n \in N \\
& \overline{\operatorname{inv}}_{j, n}+\tau_{j, n}^{+} \tilde{s d}_{j, n} \leq v_{j, n} \quad \forall j \in J, n \in N \\
& \overline{\operatorname{inv}}_{j, n}-\tau_{j, n}^{-} \tilde{s d}_{j, n} \geq 0 \quad \forall j \in J, n \in N \\
& 0 \leq v_{j, n} \leq v_{\mathrm{j}, \mathrm{n}}^{\mathrm{UB}} \quad \forall j \in J, n \in N \\
& 0 \leq p c_{m} \leq p c_{\mathrm{m}}^{\mathrm{UB}} \quad \forall m \in M \\
& p s_{m, s} \leq M U z_{m} \quad \forall j \in J, m \in M_{j}, s \in S \\
& v_{j, n} \leq v_{j, n}^{U P} \sum_{m \in M_{j}} z_{m} \quad \forall j \in J, n \in N
\end{aligned}
$$




$$
\begin{array}{ll}
\sum_{m} \mathbf{a}_{m} z_{m} \leq \mathbf{b} & \\
\delta_{j}^{s} \leq \delta_{j}^{s^{\prime}}+M \sum_{m \in M \ell \in L_{m}} z_{m} \sigma_{\ell}^{s, s^{\prime}} & \forall j \in J, s \in S, s^{\prime} \in S, s>s^{\prime},\left(s, s^{\prime}\right) \in N A \\
\delta_{j}^{s} \geq \delta_{j}^{s^{\prime}}-M \sum_{m \in M} \sum_{\ell \in L_{m}} z_{m} \sigma_{\ell}^{s, s^{\prime}} & \forall j \in J, s \in S, s^{\prime} \in S, s>s^{\prime},\left(s, s^{\prime}\right) \in N A
\end{array}
$$

Table A.1 Data for Example 1

\section{Supply of A}

$\left[10^{3}\right.$ ton / day $]$

Demand of C

[10 ton / day]

Probability of operation

Mass balance coefficient

Base capacity

[10 ton / day ]

Mean time to repair [day]

Mean time to failure

[day]
Mean $=12$

$$
\text { Std }=1
$$

Mean $=7 \quad$ Stand. Dev $=1$

Unit $1_{I}$ Unit $1_{\text {II }}$ Unit 2

Unit 3

$$
0.95
$$

0.95

0.92

0.87

$$
\alpha_{1 \mathrm{I}}=0.92
$$

$\alpha_{1 \mathrm{II}}=0.92$

$\alpha_{2}=0.85$

$\alpha_{3}=0.75$

5

5

7

9

0.25

0.25

0.25

0.25

4.75

4.75

2.88

1.67 
Table A.2 Discrete states in the superstructure of Example 1

\begin{tabular}{|c|c|c|c|c|c|}
\hline & $y_{1 I}^{s}$ & $y_{1 I I}^{s}$ & $y_{2}^{s}$ & $y_{3}^{s}$ & prob \\
\hline State 1 & 1 & 1 & 1 & 1 & 0.722 \\
\hline State 2 & 1 & 1 & 1 & 0 & 0.108 \\
\hline State 3 & 1 & 1 & 0 & 1 & 0.063 \\
\hline State 4 & 1 & 0 & 1 & 1 & 0.038 \\
\hline State 5 & 0 & 1 & 1 & 1 & 0.038 \\
\hline State 6 & 1 & 1 & 0 & 0 & 0.009 \\
\hline State 7 & 1 & 0 & 1 & 0 & 0.006 \\
\hline State 8 & 1 & 0 & 0 & 1 & 0.003 \\
\hline State 9 & 0 & 1 & 1 & 0 & 0.006 \\
\hline State 10 & 0 & 1 & 0 & 1 & 0.003 \\
\hline State 11 & 0 & 0 & 1 & 1 & 0.002 \\
\hline State 12 & 1 & 0 & 0 & 0 & $5 e-4$ \\
\hline State 13 & 0 & 1 & 0 & 0 & $5 e-4$ \\
\hline State 14 & 0 & 0 & 1 & 0 & $3 e-4$ \\
\hline State 15 & 0 & 0 & 0 & 1 & $1 \mathrm{e}-4$ \\
\hline State 16 & 0 & 0 & 0 & 0 & $3 e-5$ \\
\hline
\end{tabular}




\section{Appendix B}

\section{Scenario sampling method}

We use a discrete rate simulation model of the integrated site to obtain samples of failure times and of repair times. We use the software ExtendSim from Imagine that Inc. to build and run the model. As an output from each run we obtain a report with the type of failures that occur in the system, along with their start time and repair time. Some failures might occur multiple times, and the repair time in each instance is different since it is generated from a probability distribution. Figure B.1 shows an illustrative process network and Table B.1 the corresponding report generated after a simulation run.

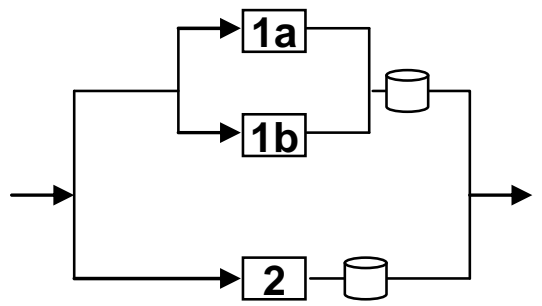

Figure B.1 Illustrative example of process network

Table B.1 Sample failure report generated during simulation run

\begin{tabular}{|c|c|c|c|c|c|c|}
\hline Event & Event time & Failure mode & $\begin{array}{c}\text { Process } \\
\text { Name }\end{array}$ & Parallel Units & TBF & TTR \\
\hline 1 & 30 & 2 & 2 & 1 & 30 & 10 \\
\hline 2 & 35 & 1 & 1 & 2 & 35 & 15 \\
\hline 3 & 55 & 2 & 2 & 1 & 25 & 20 \\
\hline 4 & 80 & 1 & 1 & 2 & 45 & 20 \\
\hline
\end{tabular}

The first column of Table B.1 enumerates the failure events. Columns 2, 3, and 4 indicate the time when the failure happened, the type of failure, and the process in which this failure occurs. In this example, there is one potential failure in each process; therefore, the failure mode and the process to which the failure refers have the same name. The columns labeled 'Parallel Units' indicates the numerical ID of units in the process that failed. We assume that a failure is equally likely in any of the units of a multiple-unit process (in the example, Process 1), and alternate successively the occurrence of the same failure mode in each of the parallel units. In this example, Event 2 occurs in Unit 1a and Event 4 in Unit 1b. Finally, the last two columns contain the time since the last failure of 
that unit and the repair time the failure will require. The repair time is sampled randomly from a distribution specified before running the simulation. Notice that the moment when the repair action is completed, it is not included as an event in the report.

The objective is to construct a sample of states from this output report. To accomplish this objective, it is necessary to keep track of which failures are occurring simultaneously in the network of Figure B.1. We keep track of the simultaneous failures by deriving Table B.2 from Table B.1.

Table B.2 Report generated by including repair events

\begin{tabular}{|c|c|c|c|c|c|c|c|c|c|}
\hline Event & Event time & Failure mode & Process & Paralle Units & TBF & TTR & RTTR 1a & RTTR 1b & RTTR 2 \\
\hline 1 & 30 & 2 & 2 & 1 & 30 & 10 & 0 & 0 & 10 \\
\hline 2 & 35 & 1 & 1 & 2 & 35 & 15 & 15 & 0 & 5 \\
\hline 3 & 40 & - & - & - & - & - & 10 & 0 & 0 \\
\hline 4 & 50 & - & - & - & - & - & 0 & 0 & 0 \\
\hline 5 & 55 & 2 & 2 & 1 & 25 & 20 & 0 & 0 & 20 \\
\hline 6 & 75 & - & - & - & - & - & 0 & 0 & 0 \\
\hline 7 & 80 & 1 & 1 & 2 & 45 & 20 & 0 & 20 & 0 \\
\hline 8 & 100 & - & - & - & - & - & 0 & 0 & 0 \\
\hline
\end{tabular}

We have added the last 3 columns labeled as 'RTTR 1a', 'RTTR 1b, and 'RTTR 2', that stand for the remaining time until repair is completed. There are also 4 new repair events in the table: Events 3, 4, 6, and 8. By keeping track of the remaining time to repair it is possible to know all failure modes that are affecting the network at any moment. The last three columns of Table B.2 can be transformed to binary format, where a 1 indicates that $R T T R=0$, and a 0 indicates that $R T T R>0$.

Table B.3 Binary table for failure modes

\begin{tabular}{|c|c|c|c|c|c|c|c|}
\hline Event & Event time & RTTR 1a & RTTR 1b & RTTR 2 & Failure mode 1a & Failure mode 1a & Failure mode 2 \\
\hline 1 & 30 & 0 & 0 & 10 & 1 & 1 & 0 \\
\hline 2 & 35 & 15 & 0 & 5 & 0 & 1 & 0 \\
\hline 3 & 40 & 10 & 0 & 0 & 0 & 1 & 1 \\
\hline 4 & 50 & 0 & 0 & 0 & 1 & 1 & 1 \\
\hline 5 & 55 & 0 & 0 & 20 & 1 & 1 & 0 \\
\hline 6 & 75 & 0 & 0 & 0 & 1 & 1 & 1 \\
\hline 7 & 80 & 0 & 20 & 0 & 1 & 0 & 1 \\
\hline 8 & 100 & 0 & 0 & 0 & 1 & 1 & 1 \\
\hline
\end{tabular}

The last three columns in Table B.3 represent a report of the discrete states of the system. Using Table B.4 to label the states, we illustrate the transition between system states in Figure B.2 
Table B.4 System states

\begin{tabular}{cccc}
\hline State & Failure mode 1a & Failure mode 1a & Failure mode 2 \\
\hline 1 & 1 & 1 & 1 \\
2 & 1 & 1 & 0 \\
3 & 1 & 0 & 1 \\
4 & 0 & 1 & 1 \\
5 & 1 & 0 & 0 \\
6 & 0 & 1 & 0 \\
7 & 0 & 0 & 1 \\
8 & 0 & 0 & 0
\end{tabular}

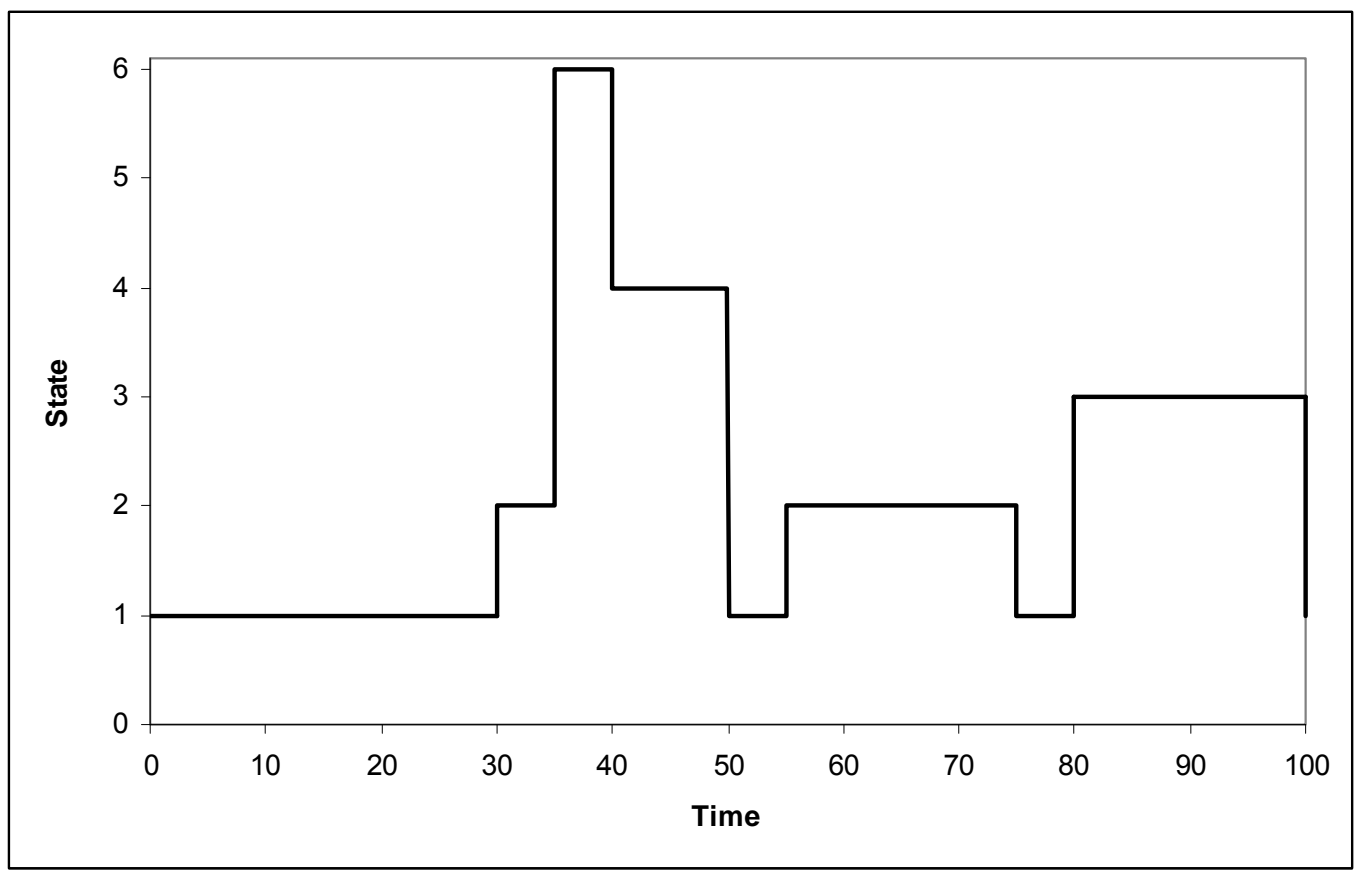

Figure B.2 Evolution of system states in one simulation run

The information in Tables B.3 and B.4 and Figure B.2 allows us to calculate the frequency of visits to each state $f r$, and the mean $m r t$ and variance $v r t$ of the distribution of residence times in each state in the sample. We can also obtain a probability prob for each state. For instance, state 1 is visited 3 times with durations of 30, 5 and 5 time units respectively. Assuming that 100 time units is a long enough horizon we can approximate the relevant parameters of the states that appear in Figure B.2. For state 1, the frequency $f r$ is 0.03 / time unit, the mean residence time mrt is 13.33 time units, and the variance of 
residence time $v r t$ is 208 [time units] ${ }^{2}$. We can also calculate the probability of state 1 as the time spent in the state divided by the duration of the operating horizon, in this case, prob $=0.40$. 